\title{
Progress in laser cooling semiconductor nanocrystals and nanostructures
}

\author{
Shubin Zhang ${ }^{1}$, Maksym Zhukovskyi², Boldizsár Jankó (D] and Masaru Kuno (D),2
}

\begin{abstract}
Over the past two decades, there have been sizable efforts to realize condensed phase optical cooling. To date, however, there have been no verifiable demonstrations of semiconductor-based laser cooling. Recently, advances in the synthesis of semiconductor nanostructures have led to the availability of high-quality semiconductor nanocrystals, which possess superior optical properties relative to their bulk counterparts. In this review, we describe how these nanostructures can be used to demonstrate condensed phase laser cooling. We begin with a description of charge carrier dynamics in semiconductor nanocrystals and nanostructures under both above gap and below-gap excitation. Two critical parameters for realizing laser cooling are identified: emission quantum yield and upconversion efficiency. We report the literature values of these two parameters for different nanocrystal/nanostructure systems as well as the measurement approaches used to estimate them. We identify $\mathrm{CsPbBr}_{3}$ nanocrystals as a potential system by which to demonstrate verifiable laser cooling given their ease of synthesis, near-unity emission quantum yields and sizable upconversion efficiencies. Feasibility is further demonstrated through numerical simulations of $\mathrm{CsPbr}_{3}$ nanocrystals embedded in an aerogel matrix. Our survey generally reveals that optimized semiconductor nanocrystals and nanostructures are poised to demonstrate condensed phase laser cooling in the near future.
\end{abstract}

\section{Introduction}

Light/matter interactions are fundamental to modern physical sciences. They open the door to manipulating the optical, chemical, and physical behavior of materials. One of the better-known optical responses of matter involves heating, wherein absorbed radiation is converted to thermal excitation of the system. This is captured by Stokes' empirical observation that the fluorescence from molecules generally occurs at lower energies than that of the excitation, the observed energy difference being transformed into molecular motion ${ }^{1}$.

Less recognized, but equally relevant, is the ability to cool matter with light. Early work by Hansch and Schawlow $^{2}$ as well as by Wineland and Dehmelt ${ }^{3}$ illustrated how light could reduce the translational motion of gases. This ultimately led to the creation of optical

\footnotetext{
Correspondence: Masaru Kuno (mkuno@nd.edu)

${ }^{1}$ Department of Physics, University of Notre Dame, Notre Dame 46556 IN, USA ¿Department of Chemistry and Biochemistry, University of Notre Dame, Notre Dame 46556 IN, USA
}

molasses $^{4-6}$, followed $\sim 10$ years later by the creation of a new quantum state of matter, a Bose-Einstein condensate, by Wieman, Cornell, and Ketterle ${ }^{7,8}$.

Today, among the remaining light/matter grand challenges is the optical cooling of condensed phases. Despite conceptual beginnings ${ }^{9}$ nearly 50 years prior to the advent of gas-phase laser cooling, condensed phase cooling remains only partially realized. This is because of significant technical hurdles that have been encountered, nearly all of which are related to material quality.

Solid/liquid state optical cooling is premised on removing thermal energy from a material through its photoluminescence. This occurs through emission at higher energies than that of the excitation and is referred to as anti-Stokes photoluminescence (ASPL). Rather than inducing translational or vibrational motion, ASPL removes thermal energy from the system, in turn cooling it. Although conceptually simple, realizing condensed phase laser cooling, in practice, represents a monumental challenge due to the delicate balance that exists between 
competing heating and cooling processes in a material. This will be discussed shortly.

Proof-of-concept condensed phase laser cooling was first demonstrated by Epstein and co-workers in 1995 using the atomic transitions of $\mathrm{Yb}^{3+}$ dopants in a fluorozirconate glass (ZBLANP:Yb $\left.{ }^{3+}\right)^{10}$. Since then, meticulous improvements in material quality and the development of $\mathrm{Yb}^{3+}$-doped yttrium lithium fluoride (YLF) crystals have enabled rare-earth-doped hosts to be cooled to successively lower temperatures ${ }^{11-15}$, ultimately reaching cryogenic temperatures in $2013^{15,16}$. These successes have been aided by improved cooling efficiencies, which stem from the narrow absorption linewidths and large absorption coefficients of $\mathrm{Yb}^{3+}$-doped YLF compared with those of ZBLANP:Yb ${ }^{3+}{ }^{16}$. Very recently, thermal payload cooling has been demonstrated with $\mathrm{Yb}^{3+}$-doped YLF, realizing a key end goal of the field ${ }^{17}$. A 2015 study by Roder et al. ${ }^{18}$ also demonstrated local cooling of liquids using optically trapped $\mathrm{Yb}^{3+}$-doped YLF nanocrystals (NCs). Figure 1 summarizes key milestones achieved in cooling rare-earth-doped solids. The dashed red line denotes the NIST (National Institute of Standards and Technology)-defined cryogenic temperature thresh$\operatorname{old}^{19}$. The corresponding blue line indicates the suggested minimum achievable temperature of $\mathrm{Yb}^{3+}$-doped YLF.

It is apparent from Fig. 1 that rare-earth-doped glasses/ crystals only partially address the challenge of cooling solids. This is because rare-earth materials are limited in their lowest achievable temperatures due to the eventual thermal depopulation of atomic ground states. Accessing temperatures below the boiling point of liquid nitrogen

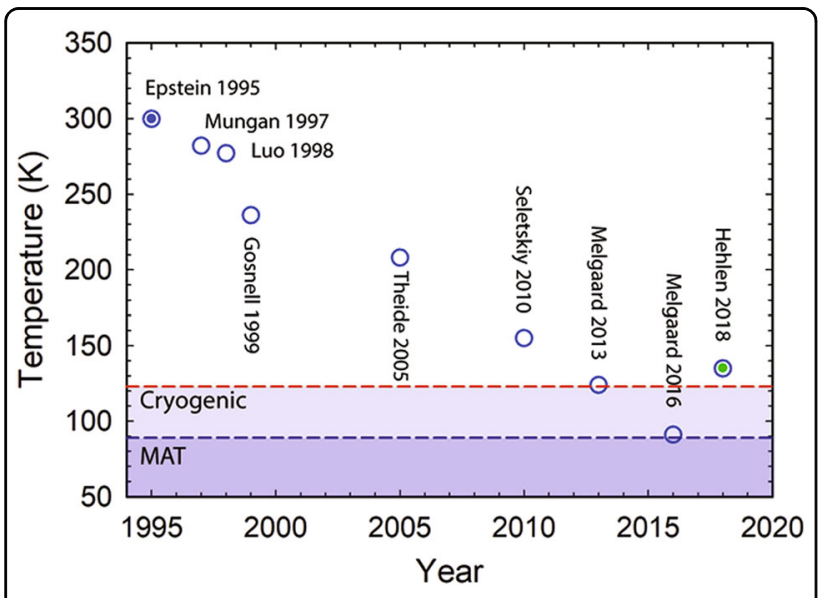

Fig. 1 Condensed phase optical cooling milestones, achieved using rare-earth-doped glasses/crystals. Data are from refs. ${ }^{10-17,19}$. Proof-of-concept and payload cooling emphasized with blue and green fills, respectively. The dashed red (blue) line denotes the NISTdefined cryogenic temperature threshold $\left(\mathrm{Yb}^{3+}\right.$-doped $\mathrm{YLF}$ minimum achievable temperature $(\mathrm{MAT})^{16}$ ) thus requires laser cooling other condensed phase systems without this intrinsic limitation.

Semiconductors possess populated valence bands, even at very low temperatures, due to their Fermi-Dirac statistics. This ensures that cooling transitions are never depleted, making possible temperature floors on the order of $10 \mathrm{~K}^{20}$. Relatively large absorption coefficients also make overall cooling efficiencies larger than those of rareearth-doped glasses/crystals. Direct integration of semiconductor optical cryocoolers into electronics is also possible given established semiconductor processing technologies. For these and other reasons, there is now significant interest in demonstrating laser cooling with semiconductors. Since 2000, much work has been done in this area on GaAs by Epstein and Sheik-Bahae, wherein near-unity external quantum efficiencies (EQEs) have been achieved through elaborate surface passivation and light management schemes ${ }^{21-23}$. Unfortunately, as with early attempts to cool rare-earth-doped glasses/crystals, sizable material quality issues have prevented any verifiable demonstration of laser cooling to date.

This review discusses progress made towards demonstrating semiconductor-based laser cooling. Of particular interest are semiconductor NCs and nanostructures due to the high likelihood of eventually achieving direct cooling with these materials. This stems from the nearly three decades of research invested in their synthesis and optical characterization $^{24,25}$, which today has resulted in an unprecedented level of control over material quality and corresponding material properties.

In the following sections, we review the optical response of semiconductor NCs and nanostructures to both abovegap (Stokes) and below-gap (anti-Stokes) excitation. We then describe potential mechanisms leading to ASPL and discuss the critical $\mathrm{NC} /$ nanostructure parameters required to realize laser cooling. The accompanying tables provide literature-compiled estimates of these parameters. We end with cooling simulations of $\mathrm{CsPbBr}_{3} \mathrm{NCs}$, which we have identified as a promising system by which to demonstrate laser cooling. For those interested, comprehensive reviews of general condensed phase laser cooling can be found in the following refs. ${ }^{23,26-29}$ and monographs ${ }^{30,31}$.

\section{A delicate balance}

What makes cooling a semiconductor so difficult? Realizing condensed phase laser cooling rests on achieving a delicate balance between competing cooling and heating processes in a material. This can be understood qualitatively since cooling is premised on removing a few quanta of thermal energy from a system during each cycle of excitation and subsequent emission. To put this into context, phonon energies in semiconductors range from 25 to $44 \mathrm{meV}^{32-34}$. By contrast, competing nonradiative 
recombination processes introduce what is essentially the material's entire band gap energy as heat into the lattice. Typical band gap energies range from 1 to $4 \mathrm{eV}^{32,35}$. This highlights the uncomfortable reality that, given the close to two orders of magnitude difference between cooling and heating energies, cooling occurs only when radiative recombination is the near-exclusive carrier recombination process in a material.

A semiconductor's EQE thus dictates whether optical cooling can be achieved. In semiconductor nanostructures, EQE is synonymous with the emission quantum yield (QY), as photon trapping and reabsorption processes are negligible due to the small dimensions of the samples. Near-unity QYs are therefore primarily restricted by competing nonradiative processes. This places stringent restrictions on material quality and makes much of semiconductor laser cooling a matter of materials optimization, as neither the basic principles of laser cooling nor its possibility is disputed ${ }^{36}$.

\section{Early work on semiconductors}

Early work to demonstrate semiconductor optical cooling focused on GaAs, a mature system in terms of its growth and subsequent processing. Preventing the realization of near-unity EQEs in GaAs, however, were two key problems. The first stemmed from low overall internal QYs due to the presence of surface recombination. This was ultimately addressed by developing various growth ${ }^{37}$ and surface passivation schemes ${ }^{22}$ that effectively removed undesired carrier recombination channels.

The second involved light trapping and subsequent emission reabsorption (also referred to as photon recycling). This scenario arises from the large refractive index difference that exists at the interface between a semiconductor and its surrounding medium (e.g., air). Light management schemes are therefore needed to achieve near-unity EQEs even if internal QYs are high. In practice, this has entailed introducing index matching, light extraction elements to the semiconductor surface ${ }^{21,38}$.

Only two studies have attempted the optical cooling of GaAs or any other bulk semiconductor. The first by Gauck et al. ${ }^{38}$ involved a GaAs/GaInP double heterostructure with an impressive 96\% EQE. To achieve this, GaInP layers were first used to passivate GaAs surface states. The passivation layer additionally aided in index matching the GaAs active layer to a hemispherical $\mathrm{ZnSe}$ light extraction dome.

Despite these optimization efforts, only net heating was observed when the excitation laser was tuned to the red of the mean emission wavelength. It was suggested that if the laser could be further detuned while maintaining an optimal excitation intensity, then cooling could be realized given the absence of any apparent parasitic absorption in the system.
The second study by Bender et al. ${ }^{21}$ likewise focused on GaAs/GaInP double heterostructures but now with a record $99.5 \% \mathrm{EQE}$. This was achieved by more carefully optimizing one of two GaAs/GaInP interfaces using a stress-relieving GaP intermediary layer. As with Gauck, a $(\mathrm{ZnS})$ light extraction hemisphere was added to maximize emission out-coupling. Yet, despite having a record EQE, no net cooling was observed. It was therefore suggested that cooling had been prevented by parasitic sub-band gap absorption of the incident light, leading to heating. Although the origin of this parasitic absorption was not identified, speculation centered on either GaAs/GaInP interface states or native point defects (vacancies, interstitials) within the GaInP passivation layer.

\section{Towards nanostructures}

While bulk semiconductors can display near-unity EQEs, it is evident that complex processing is required. Furthermore, achieving these near-unity values is far from routine. For these reasons, increasing attention has focused on semiconductor nanostructures, which possess notable advantages over their bulk counterparts. This includes the absence of light trapping/reabsorption effects due to their small physical dimensions as well as higher (as made) QYs, the latter occurring despite their large surface-to-volume ratios.

To illustrate these points, consider CdSe, the prototypical colloidal NC system ${ }^{39}$, which takes typical diameters between $2-12 \mathrm{~nm}^{40}$. These NCs effectively behave as dipole emitters ${ }^{41}$. Colloidal NCs also possess sizable QYs, as demonstrated by systems such as $\mathrm{PbSe}$ $(\mathrm{QY} \sim 85 \%)^{42}$ and, more recently, by $\mathrm{CsPbBr}_{3}$ (QY $\sim 50$ $-90 \%)^{43}$. Additional surface passivation schemes, which produce core/shell $\mathrm{NCs}^{44-46}$, now frequently yield nearunity QYs. When coupled to their facile chemistries as well as tunable band gaps, colloidal NCs and associated nanostructures represent obvious systems by which to demonstrate condensed phase laser cooling.

Perhaps, the most compelling reason why semiconductor nanostructures have attracted interest today is the recent report by Xiong and co-workers ${ }^{47-49}$, suggesting the cooling of individual CdS nanobelts (NBs). Although CdS is not the first system to come to mind when contemplating condensed phase laser cooling, the possibility of high purity/low background parasitic absorption, low carrier mobilities, low associated surface recombination velocities, and smaller Auger coefficients make it an intriguing system to investigate. Adding to this, Xiong and co-workers ${ }^{50}$ have suggested the cooling of individual hybrid perovskite nanoplatelets. An additional report by Fontenot et al. ${ }^{51,52}$ has likewise suggested the optical cooling of commercial, overcoated CdSe NCs. Because of these tantalizing hints that semiconductor nanostructures can be cooled, the remainder of this 
review focuses on explaining emission upconversion in colloidal semiconductor $\mathrm{NCs}$ and associated nanostructures, as well as illustrating the underlying hinderances to achieving laser-induced cooling.

\section{Semiconductor NC/nanostructure upconversion}

Two common processes exist for upconverting light in isolated semiconductor nanostructures. They involve the one- or two-photon absorption of light. The former are relevant to condensed phase laser cooling, while the latter are not. This is because one-photon processes, which use sub-band gap photons, necessarily involve semiconductor lattice phonons to upconvert the initial excitation. In turn, thermal energy is removed from the system and can lead to net cooling. By contrast, two-photon processes bypass phonon involvement since the energy of two subgap photons readily spans the semiconductor band gap.

In general, within one- or two-photon processes, distinctions arise due to the identity of the intermediate state, whether real or virtual. Real states are often defectrelated and have lifetimes on the order of nanoseconds. Virtual states, by contrast, possess significantly shorter lifetimes, dictated by time/energy uncertainty. This makes upconversion involving the former much more likely under conditions where excitation intensities are low.

Most existing nanostructure upconversion studies suggest the involvement of real, defect-related intermediate states that are relatively "dark" (i.e., low oscillator strength) in absorption. This includes work on $\mathrm{TiO}_{2}{ }^{53,54}$, $\mathrm{CdS}^{55-57}, \mathrm{CdSe}^{58-62}, \mathrm{CdTe}^{59,63-66}, \mathrm{InP}^{55,58}, \mathrm{PbS}^{67}, \mathrm{Ag}_{2} \mathrm{~S}^{68}$, and $\mathrm{CsPbBrI}_{2} \mathrm{NCs}^{69}$. A few suggest the involvement of virtual intermediate states, especially when two-photon upconversion has been claimed (e.g., in $\mathrm{CdS}^{70}$ and $\mathrm{CdTe}^{71}$ $\mathrm{NCs}$ ). Others (e.g., $\mathrm{PbS}^{72}$ and $\mathrm{CsPbBr}_{3}{ }^{73}$ ) leave open the possibility of another upconversion mechanism, as of yet undefined.

Figure 2 schematically illustrates one-photon processes involving virtual (Fig. 2a) and real (Fig. 2b) intermediate states. For either, transitions between $\mathrm{NC}$ valence and conduction band states occur with $G^{\prime}$, the subgap excitation rate, associated with an incident (subgap or antiStokes) excitation intensity $I_{\text {exc }}$. Relevant conduction and valence band state carrier densities are denoted in the figure by $n$ and $p$, respectively. Upconversion occurs through interaction with lattice phonons (illustrated with an associated rate constant, $k_{\mathrm{ph}}$ ) and ultimately leads to higher energy ASPL with an intensity $I_{\mathrm{ASPL}}$ (illustrated using an associated rate constant, $k_{\mathrm{r}}$ ). For simplicity, Fig. $2 \mathrm{~b}$ assumes that the ASPL adopts the same energy as the normal band edge emission of the material and originates from the same emitting state.

In the case where a real intermediate state is involved, carrier retrapping becomes possible. This is denoted in Fig. $2 \mathrm{~b}$ using the associated rate constant $k_{\mathrm{t}}$. Similarly, radiative or nonradiative relaxation from the intermediate state back to the system's ground state is also possible and is denoted with the associated rate constant $k_{\mathrm{p}}$. The former results in an energetically distinct, defect-related contribution to the material's overall emission.

Figure 3 illustrates associated two-photon upconversion processes involving virtual or real intermediate states. These processes occur under significantly higher excitation intensities and are characterized by a quadratic ASPL $I_{\text {exc }}$ dependency due to their two-photon nature (i.e., $I_{\mathrm{ASPL}} \propto I_{\mathrm{exc}}^{2}$ ). When a real state is involved, the process is sometimes referred to as a two-step, two-photon upconversion due to the fact that the intermediate state possesses a finite lifetime. Of note is the absence of any phonon involvement, making two-photon processes nominally irrelevant to achieving condensed phase laser cooling.

An additional emission upconversion mechanism exists in nanostructures and stems from the Auger-induced recombination of photogenerated carriers. Auger recombination generally occurs at very large excitation intensities and involves the resonant excitation of multiple electron-hole pairs ${ }^{74,75}$. Following thermalization, energy transfer between recombining electron-hole pairs and
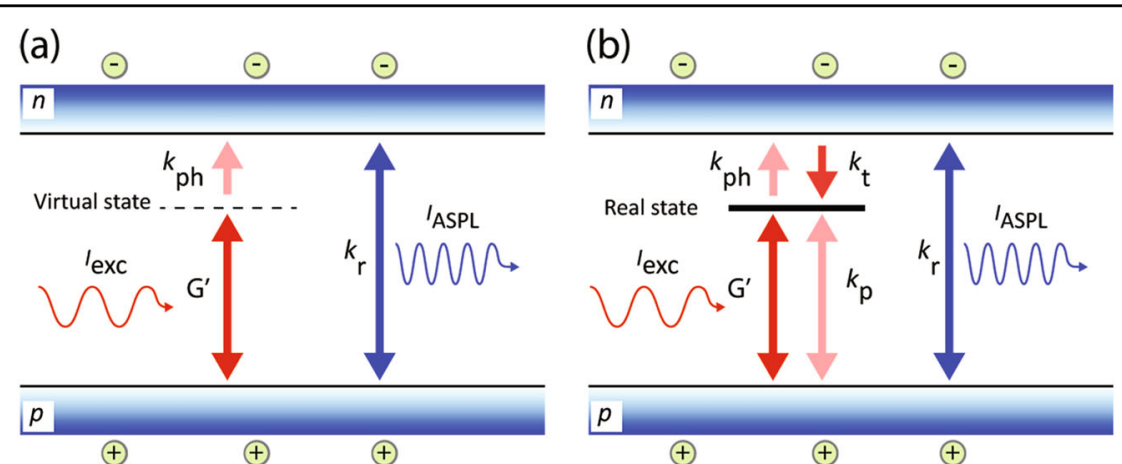

Fig. 2 Schematic illustrations of one-photon/phonon upconversion. a real state- and $\mathbf{b}$ virtual state-mediated 

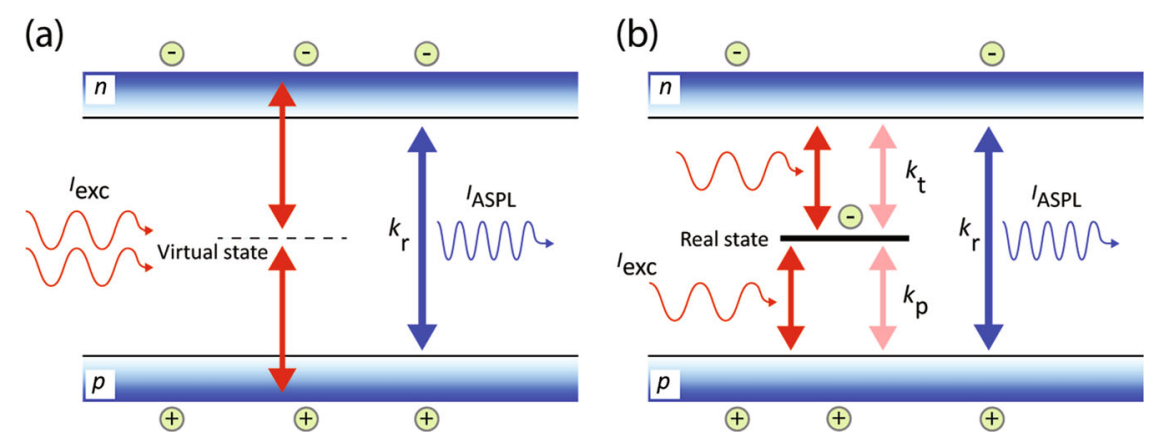

Fig. 3 Schematic illustration of two-photon upconversion. a real state- and $\mathbf{b}$ virtual state-mediated

other resident electron-hole pairs leads to carrier promotion into higher energy states, either within or associated with the nanostructure. Assuming that radiative recombination is possible from these states, upconverted emission results.

There are several points worth noting: (a) As with twophoton processes, Auger-induced emission upconversion lacks phonon involvement. (b) Under anti-Stokes excitation conditions, intermediate states must be real and must have sizable absorption cross-sections if multiple electron-hole pairs are to be generated. These states are not necessarily dark. (c) Given that involved higher energy states are generally extrinsic to the system being studied, they will be absent in isolated NCs/nanostructures. To illustrate, Si NC networks exhibit Auger-induced upconversion where interparticle energy "ladder climbing" occurs due to Auger-induced charge transfer into neighboring NCs with larger band gaps ${ }^{75}$. These Augerinduced upconversion processes will be absent if individual NCs are studied.

\section{$\mathrm{NC} /$ nanostructure photophysics under above gap excitation}

Given their relevance to laser cooling, we now focus on one-photon/phonon upconversion processes in NCs and nanostructures. Before discussing critical parameters required to achieve cooling, we review nanostructure photophysics under above gap (i.e., Stokes) excitation. Of particular importance is the emission QY of the material, as this is key to whether cooling can be achieved.

In NCs and nanostructures, QYs are primarily dictated by the existence of competing nonradiative recombination processes. Consequently, for laser cooling, it is important to measure QYs as well as understand their dependence on $I_{\text {exc }}$, as this contains information about competing carrier recombination processes. In NCs/nanostructures, the dependency can be assessed by examining the behavior of their emission intensity $\left(I_{\mathrm{em}}\right)$ as a function of $I_{\mathrm{exc}}$ since competing recombination mechanisms cause predictable changes to the expected power-law growth of $I_{\mathrm{em}}$.
Specifically, it can be shown that $I_{\mathrm{em}} \propto I_{\text {exc }}^{b}$ with an observed power law growth exponent, $b$, that varies, depending on the dominant carrier recombination process occurring in a $\mathrm{NC} /$ nanostructure at a given excitation intensity $^{76,77}$. Of specific interest are carrier trapping events, which ultimately suppress QYs.

The following equations model $I_{\mathrm{em}}$ under above gap excitation:

$$
\begin{aligned}
& \frac{\mathrm{d} n}{\mathrm{~d} t}=G-k_{\mathrm{r}} n p-k_{\mathrm{t}} n\left(N_{\mathrm{t}}-n_{\mathrm{t}}\right)+k_{\mathrm{ph}} n_{\mathrm{t}}-k_{\text {Auger }} n p^{2} \\
& \frac{\mathrm{d} p}{\mathrm{~d} t}=G-k_{\mathrm{r}} n p-k_{\mathrm{p}} n_{\mathrm{t}} p-k_{\text {Auger }} n p^{2} \\
& \frac{\mathrm{d} n_{\mathrm{t}}}{\mathrm{d} t}=k_{\mathrm{t}} n\left(N_{\mathrm{t}}-n_{\mathrm{t}}\right)-k_{\mathrm{ph}} n_{\mathrm{t}}-k_{\mathrm{p}} n_{\mathrm{t}} p
\end{aligned}
$$

In Eqs. $1-3, G$ is the carrier generation rate, $n(p)$ is the photogenerated electron (hole) carrier density, $k_{\mathrm{r}}$ is a bimolecular radiative recombination rate constant, $k_{\mathrm{t}}$ is a bimolecular trapping rate constant (the existence of electron traps is assumed for convenience), $k_{\mathrm{ph}}=k_{\mathrm{ph}}^{\mathrm{o}} e^{-\frac{\Delta E}{k T}}$ is a trap state depopulation rate constant, with $k_{\mathrm{ph}}^{\mathrm{o}}$ being a detrapping attempt frequency and $\Delta E$ being the trap depth, $k_{\mathrm{p}}$ is a trap recombination rate constant, and $k_{\text {Auger }}$ is the Auger rate constant. $N_{\mathrm{t}}$ is the native trap density of the material, with $n_{\mathrm{t}}$ being the occupied density. Implicit in the model is that the material is intrinsic. Figure 4 summarizes the kinetic model, leaving out Auger processes for simplicity.

At very low excitation intensities where trapping dominates and where $N_{\mathrm{t}} \gg n_{\mathrm{t}}$, an approximate analytical solution to the model yields the following emission intensity:

$$
I_{\mathrm{em}} \cong k_{\mathrm{r}} \frac{\left(G+k_{\mathrm{ph}} n_{\mathrm{t}}\right)}{k_{\mathrm{t}} N_{\mathrm{t}}} \frac{G}{k_{\mathrm{p}} n_{\mathrm{t}}} .
$$

Equation 4 shows that $I_{\mathrm{em}}$ grows in a power-law fashion with a slope between 2 and 1, depending on the rate constants involved. At higher intensities, prior to the 


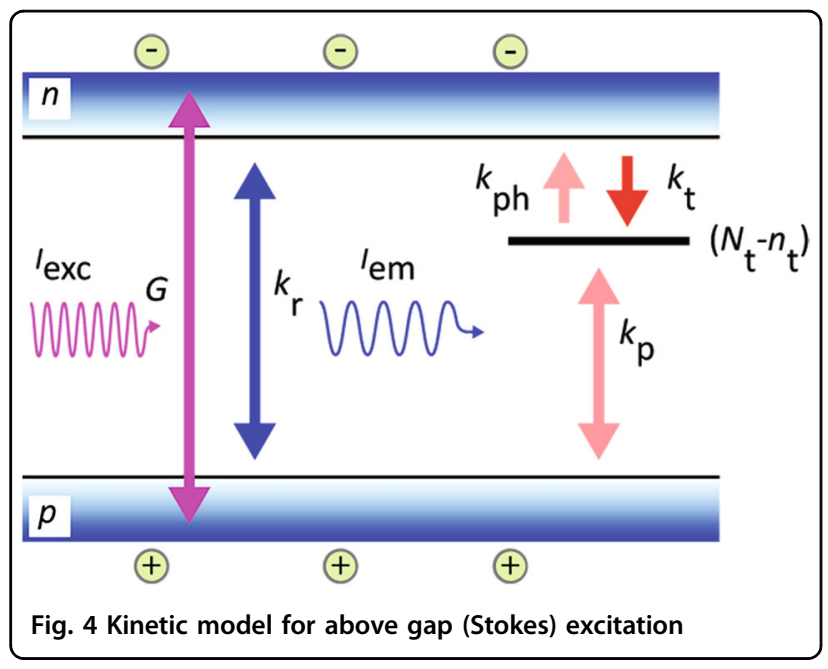

onset of significant Auger recombination and where traps saturate, it can similarly be shown that $I_{\mathrm{em}} \propto I_{\mathrm{exc}}$ (i.e., $b \sim 1$ ).

Figure 5 shows exact numerical solutions to Eqs. 1-3 using rate constants and parameters established for $\mathrm{CdS}$ $\mathrm{NBs}^{57}: \quad k_{\mathrm{r}}=9.55 \times 10^{-12} \mathrm{~cm}^{3} \mathrm{~s}^{-1}, \quad k_{\mathrm{t}}=6.31 \times 10^{-11} \mathrm{~cm}^{3}$ $\mathrm{s}^{-1}, k_{\mathrm{p}}=10^{-11} \mathrm{~cm}^{3} \mathrm{~s}^{-1}, k_{\text {Auger }}=10^{-30} \mathrm{~cm}^{6} \mathrm{~s}^{-1}, k_{\mathrm{ph}}^{\mathrm{o}}=10^{4}$ $\mathrm{s}^{-1}$, and $T=300 \mathrm{~K}$. Figure 5a shows the expected power law growth of $I_{\mathrm{em}}$ and illustrates its $I_{\mathrm{exc}}$ dependency for different $N_{\mathrm{t}}$. Figure $5 \mathrm{~b}$ highlights specific variations of the power-law growth exponent for $N_{\mathrm{t}}=10^{16} \mathrm{~cm}^{-3}$. Notably, at low $I_{\text {exc }}, b$ adopts a value between 1 and 2, following the qualitative prediction of Eq. 4. At higher intensities, where bimolecular radiative recombination, as opposed to carrier trapping, dominates, $b$ approaches 1 . This again agrees with the approximate analytical solution to Eqs. 13 under conditions where bimolecular radiative recombination dominates all other processes. The condition is additionally associated with a maximum in the emission QY. At even higher intensities, Auger recombination causes $b<1$. Figure $5 \mathrm{c}$ shows the associated QYs for all $N_{\mathrm{t}}$ values and reveals peaked functions, which first grow with $I_{\text {exc }}$, but then decrease due to the Auger-induced quenching of excitations.

To illustrate that these trends are borne out experimentally, Fig. 6 shows results acquired from a single methylammonium lead iodide $\left(\mathrm{MAPbI}_{3}\right)$ crystal. Figure $6 \mathrm{a}$ reveals that $I_{\mathrm{em}}$ grows in a power-law fashion with the $b$ values depending on $I_{\mathrm{exc}}$. As seen earlier and as predicted by Eq. $4, b$ lies between 2 and 1 . Increasing $I_{\text {exc }}$ causes $b$ to decrease towards 1 . Beyond this, $b$ grows sublinearly due to the onset of Auger recombination. These trends all match the results shown in Fig. 5.

Figure $6 \mathrm{~b}$ plots the $I_{\text {exc }}$ dependence of the normalized emission intensity $\frac{I_{\text {em }}}{I_{\text {exc }}}$, which is proportional to QY. $\frac{I_{\text {em }}}{I_{\text {exc }}}$ peaks much like the QYs in Fig. 5c. As will be demonstrated shortly when discussing a QY estimation technique called power-dependent photoluminescence (PDPL),

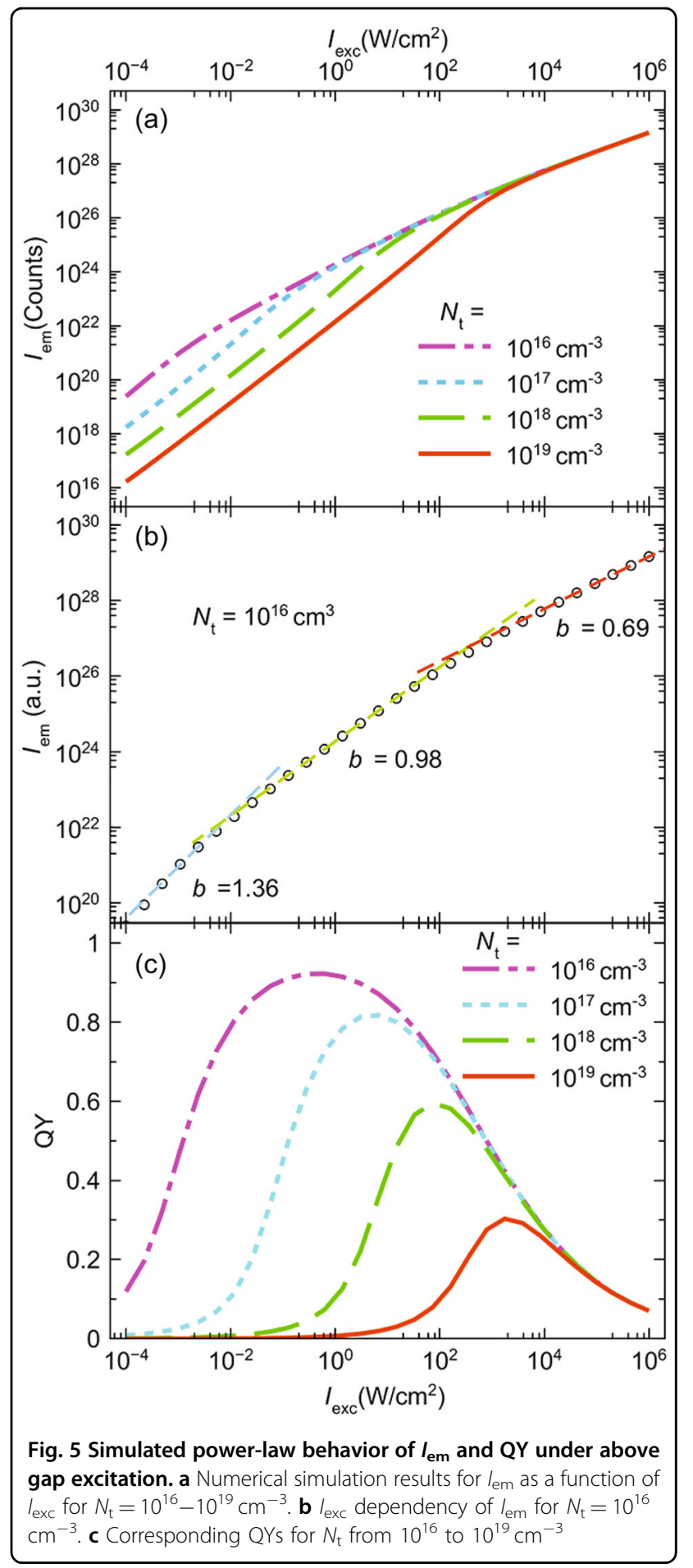

$\frac{I_{\mathrm{em}}}{I_{\mathrm{exc}}}$ can be fit with the results of a kinetic model to extract an absolute optimal QY. For the $\mathrm{MAPbI}_{3}$ sample shown here, a maximum QY of $79 \%$ is found.

\section{A critical QY exists to achieve laser cooling}

A critical $\mathrm{NC} /$ nanostructure $\mathrm{QY}\left(\mathrm{QY}_{\text {crit }}\right)$, required to realize laser cooling, emerges from the energy balance 
between competing cooling and heating processes in the $\mathrm{NC} /$ nanostructure. To explicitly demonstrate this, the

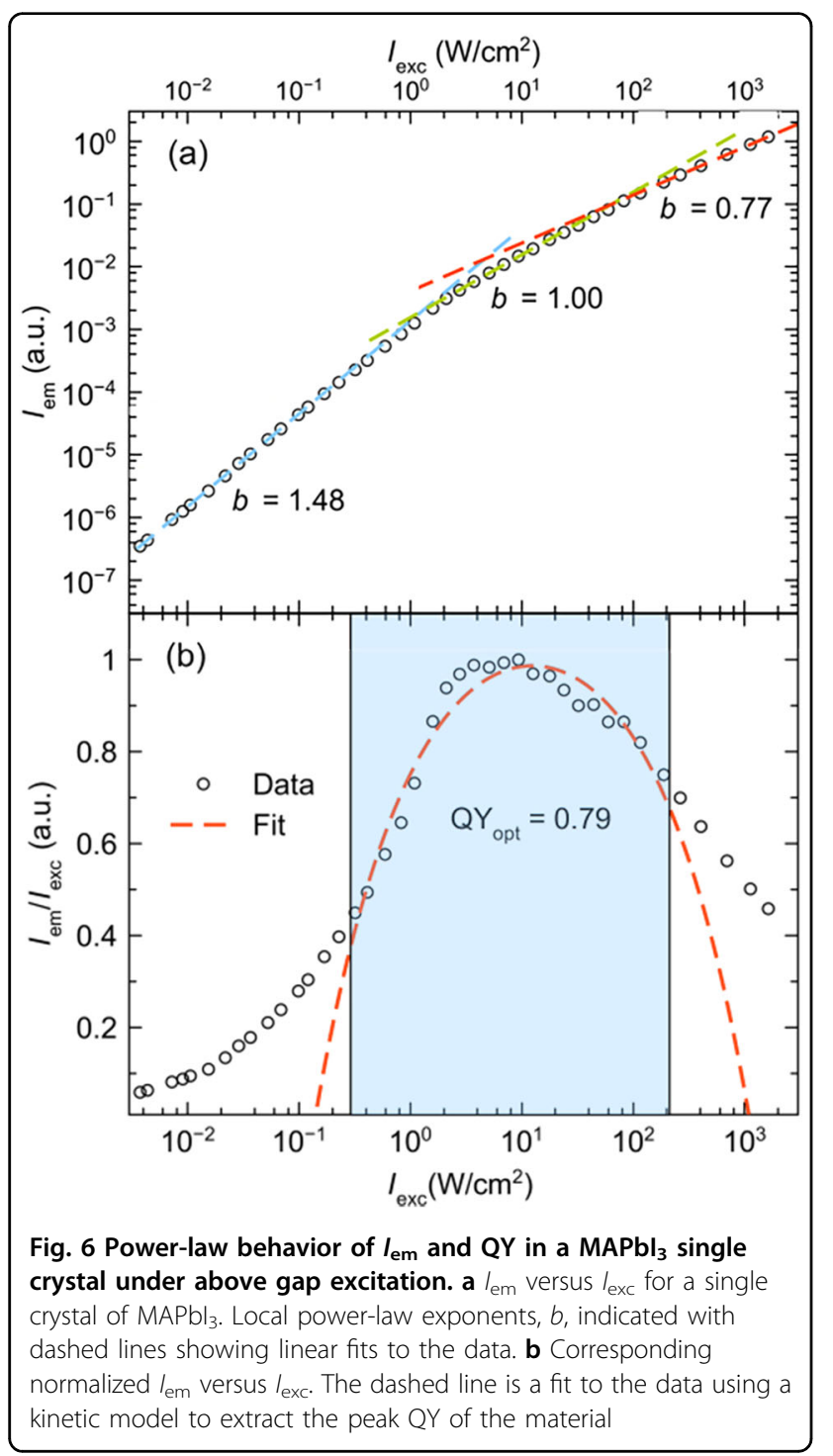

thermal energy removed via ASPL can be expressed as $Q_{\text {cool }}=M \eta_{\mathrm{ASPL}} \mathrm{QY} \Delta E$, where $M$ is the number of induced sub-band gap excitations, $\Delta E$ is the energetic detuning of the laser into the semiconductor gap, and $\eta_{\mathrm{ASPL}}$ is the fraction of those excitations promoted to the $\mathrm{NC} /$ nanostructure band edge.

The corresponding thermal energy introduced into the system due to nonradiative relaxation is $Q_{\text {heat }}=$ $M \eta_{\mathrm{ASPL}}(1-\mathrm{QY})\left(E_{\mathrm{g}}-\Delta E\right)+M\left(1-\eta_{\mathrm{ASPL}}\right) f\left(E_{\mathrm{g}}-\Delta E\right)$,

where the first term reflects the fraction of upconverted excitations that recombine nonradiatively and where the second reflects the fraction $(f)$ of excitations not upconverted, which also recombine nonradiatively. The upconversion efficiency, $\eta_{\mathrm{ASPL}}$, is therefore an important metric for laser cooling since it quantifies the balance between cooling and heating in a material together with QY. Optimal laser cooling conditions require large $\eta_{\text {ASPL }}$ values.

A critical QY to achieve cooling emerges when $Q_{\text {cool }}=$ $Q_{\text {heat }}$. What results is

$$
Q Y_{\text {crit }}=\left(\frac{E_{\mathrm{g}}-\Delta E}{E_{\mathrm{g}}}\right)\left[\frac{\eta_{\mathrm{ASPL}}+\left(1-\eta_{\mathrm{ASPL}}\right) f}{\eta_{\mathrm{ASPL}}}\right] .
$$

To put $\mathrm{QY}_{\text {crit }}$ into context, Table 1 lists $\mathrm{QY}$ crit values calculated for various semiconductors, including $\mathrm{CdS}$, when $\Delta E=25$ and $100 \mathrm{meV}$. For comparison purposes, the bulk longitudinal optical phonon energy $\left(\hbar \omega_{\mathrm{LO}}\right)$ of each semiconductor has been provided. In all cases, an upconversion efficiency of $\eta_{\mathrm{ASPL}}=1$ is assumed to illustrate the minimum limiting value for $\mathrm{QY}_{\text {crit }}$.

Table 1 reveals general trends regarding the likelihood of achieving optical cooling with a given $\mathrm{NC} /$ nanostructure system. Namely, whereas quantum-confinementinduced band gap shifts are important for many $\mathrm{NC} /$ nanostructure applications, they do not represent an advantage here since larger band gaps simply increase $\mathrm{QY}_{\text {crit. }}$ This reduces the margin of error for realizing optical cooling. By the same token, NC systems with

Table 1 Critical NC QYs to achieve optical cooling

\begin{tabular}{|c|c|c|c|c|c|c|}
\hline System & $\begin{array}{l}\text { Diameter or edge } \\
\text { length }(\mathrm{nm})\end{array}$ & $E_{\mathrm{g}}(\mathrm{eV})$ & $\hbar \omega_{\mathrm{LO}}(\mathrm{meV})$ & $\begin{array}{l}\mathrm{QY}_{\text {crit }}(\%) \Delta E=25 \mathrm{meV} \\
=1\end{array}$ & $\begin{array}{l}\mathrm{QY}_{\text {crit }}(\%) \Delta E=100 \mathrm{meV} \\
=1\end{array}$ & $\begin{array}{l}\text { Sizing, } E_{\mathrm{g}} \text {, and } \\
\hbar \omega_{\text {Lo }} \text { refs. }\end{array}$ \\
\hline CdS NC & $1.3-5.5$ & $4.74-2.81$ & 37.8 & $99.5-99.1$ & $97.9-96.4$ & 32,125 \\
\hline CdSe NC & $1.0-8.5$ & $3.57-1.91$ & 26.3 & $99.3-98.7$ & $97.2-94.8$ & 32,125 \\
\hline CdTe NC & $3.5-9.0$ & $2.15-1.67$ & 20.8 & $98.8-98.5$ & $95.3-94.0$ & 32,125 \\
\hline $\mathrm{PbS} N \mathrm{C}$ & $4.0-8.5$ & $1.23-0.67$ & 25.4 & $98.0-96.3$ & $91.9-85.1$ & 32,126 \\
\hline PbSe NC & $3.0-5.5$ & $1.26-0.73$ & 16.5 & $98.0-96.6$ & $92.1-86.3$ & 32,127 \\
\hline $\mathrm{CsPbBr}_{3} \mathrm{NC}$ & $4.0-12.5$ & $2.70-2.41$ & 20.5 & $99.1-99.0$ & $96.3-96.0$ & 128,129 \\
\hline $\mathrm{CsPb}_{3} \mathrm{NC}$ & $3.4-12.5$ & $2.13-1.85$ & NA & $98.8-98.6$ & $95.3-94.6$ & 130 \\
\hline
\end{tabular}


smaller starting bulk band gaps possess lower overall $\mathrm{QY}_{\text {crit }}$ values. This means that, all things being equal, $\mathrm{CdTe}$ should be easier to cool than CdS. In practice, however, the ease and robustness of achieving near-unity QYs in a material will ultimately dictate its application to laser cooling. Finally, larger $\Delta E$ values lower $\mathrm{QY}_{\text {crit, }}$ which suggests that $\Delta E$ should be made as large as possible. However, upconversion efficiencies scale inversely with $\Delta E$ and, in principle, follow the Boltzmann distribution, $e^{-\frac{\Delta E}{k T}}$. Consequently, a limit exists to how large $\Delta E$ can be before $\eta_{\mathrm{ASPL}}$ is adversely affected.

One last consideration exists regarding $Q Y_{\text {crit. }}$ Table 1 lists conservative values based on the apparent band gaps of NCs/nanostructures. The existence of a Stokes shift $\left(\Delta E_{\text {Stokes }}\right)$ between the absorption and emission ${ }^{78}$, however, means that critical QYs will be slightly reduced in practice. Stokes shifts generally arise from weak oscillator strength, band edge emitting states intrinsic to $\mathrm{NCs} /$ nanostructures. This is evidenced by size-dependent $\Delta E_{\text {Stokes }}$ values, which range from $\sim 10$ to $\sim 100 \mathrm{meV}^{78,79}$.

Since the upconverted emission originates from the $\mathrm{NC} /$ nanostructure emitting state, $Q_{\text {heat }}$ can be reexpressed as $Q_{\text {heat }}=M \eta_{\mathrm{ASPL}}(1-\mathrm{QY})\left(E_{\mathrm{em}}-\Delta E\right)+M$ $\left(1-\eta_{\mathrm{ASPL}}\right) f\left(E_{\mathrm{em}}-\Delta E\right)$, where $E_{\mathrm{em}}=E_{\mathrm{g}}-\Delta E_{\text {Stokes. }}$. A $\mathrm{QY}_{\text {crit }}$ that accounts for $\mathrm{NC} /$ nanostructure Stokes shifts is therefore

$$
\mathrm{QY}_{\mathrm{crit}}=\left(\frac{E_{\mathrm{em}}-\Delta E}{E_{\mathrm{em}}}\right)\left[\frac{\eta_{\mathrm{ASPL}}+\left(1-\eta_{\mathrm{ASPL}}\right) f}{\eta_{\mathrm{ASPL}}}\right],
$$

with $\Delta E$ redefined as the energy difference between excitation and emission. Using Eq. 6, entries for the $\mathrm{CsPbBr}_{3} \mathrm{NCs}$ in Table 1 decrease slightly to 99.0 and 96.2-95.8 for $\Delta E=25$ and $100 \mathrm{meV}$, respectively.

\section{How are NC/nanostructure QYs estimated?}

We now review approaches by which $\mathrm{NC} /$ nanostructure QYs are quantified in practice. This is motivated by the need to obtain accurate estimates as QY values approach unity. As will be seen, experimental QY measurements generally involve relative, absolute, and calorimetric approaches.

\section{Relative QYs}

Perhaps, the most popular way to measure NC/nanostructure QYs is to compare their integrated emission intensities to that of a known QY reference (usually an organic dye) under identical measurement conditions. The QY can then be estimated using

$$
\mathrm{QY}=\mathrm{QY} \text { ref }\left(\frac{I_{\mathrm{s}}}{I_{\text {ref }}}\right)\left(\frac{A_{\text {ref }}}{A_{\mathrm{s}}}\right)\left(\frac{n_{\text {solvent }}}{n_{\text {ref }}}\right)^{2},
$$

where $\mathrm{QY}$ ref is the reference QY, $I_{\mathrm{s}}\left(I_{\mathrm{ref}}\right)$ is the integrated emission intensity of the sample (reference), and $n_{\text {solvent }}$ $\left(n_{\text {ref }}\right)$ is the corresponding refractive index of the solvent in which the $\mathrm{NC} /$ nanostructure (reference) is dissolved. The fraction of light absorbed by the sample and reference at their respective excitation wavelengths, $A_{\mathrm{s}}$ and $A_{\text {ref, }}$ is determined using $A=1-10^{-\mathrm{Abs}}$, where Abs is the sample/reference absorbance at the excitation wavelength used to induce emission.

Relative QY measurements can be conducted using conventional absorption and emission spectrometers. This simplicity, however, belies a number of uncertainties, which complicate accurate QY measurements. Specifically, errors stemming from the instrument response or associated with both the reference and sample must be accounted for if accurate results are to be obtained.

Regarding instrument-related issues, $\mathrm{NC} /$ nanostructure and reference emission spectra must be corrected for the wavelength-dependent spectral responsivity of absorption/emission spectrometers. Furthermore, if different sample and reference excitation wavelengths are used, the frequency-dependent response of the excitation source must be accounted for to eliminate any differences in the incident photon flux ${ }^{80,81}$. In principle, these instrumentrelated issues can be mitigated by using a suitable reference that absorbs and emits in the same wavelength region as that of the $\mathrm{NC} /$ nanostructure ${ }^{82}$.

It is evident that the choice of reference critically dictates the accuracy of relative QYs. Reference selection can be particularly challenging in the ultraviolet $(<400 \mathrm{~nm})$ and near-infrared $(>750 \mathrm{~nm})$ regimes ${ }^{83}$. This is because the reported QYs for dyes in these spectral windows vary greatly. To illustrate, the reported QY for blue-emitting Coumarin $\mathrm{C} 153$ ranges from 26 to $58 \%^{80}$. Red-emitting Cresyl violet has a reported QY that ranges from 51 to $67 \%^{83}$. Even well-characterized dyes such as Rhodamine $6 \mathrm{G}$ do not have consistent QYs in the literature. For Rhodamine 6G, reported QYs range from 88 to $95 \%{ }^{82}$. Moreover, the use of a reference having a disparate QY relative to the sample can introduce estimation errors, especially if the sample pushes the instrument's lower limit of detection. Obtaining accurate relative QYs therefore restricts reference dyes to those having nearidentical absorption and emission wavelengths as well as QYs to the NC/nanostructure being probed ${ }^{80}$.

Beyond the choice of reference, the employed dye concentration can introduce measurement error. Too high a concentration results in emission reabsorption (also called the inner filter effect), which decreases QYs. Conversely, low concentrations become problematic for many colloidal $\mathrm{NC}$ /nanostructure samples due to the irreversible loss of surface passivating agents under dilute conditions. This results in both decreased emission QYs and sample instabilities that lead to eventual precipitation $^{84-86}$. Furthermore, polarization sensitivities 
associated with anisotropic nanostructures as well as potential light scattering effects complicate measurements $^{87,88}$. Finally, solvent light scattering and (possibly) fluorescence contribute to measurement errors ${ }^{85,89}$. It is thus evident that relative QY measurements, while simple, do not represent the most robust approach by which to estimate absolute $\mathrm{NC} /$ nanostructure QYs.

\section{Absolute QY measurements Integrating sphere}

Absolute QY estimates are generally made by directly measuring a sample's ratio of emitted to absorbed photons. As no dye reference is involved, the abovehighlighted reference issues are obviated. Intensity losses due to scattering and sample anisotropies are also eliminated. In practice, absolute QY measurements entail using an integrating sphere in which QYs are estimated using

$$
\mathrm{QY}=\frac{I_{\mathrm{em}}}{I_{\text {exc,ref }}-I_{\text {exc }, \mathrm{s}}} .
$$

In Eq. 8, $I_{\mathrm{em}}$ is the integrated emission intensity of the sample under direct illumination, while $I_{\text {exc,s }}\left(I_{\text {exc,ref }}\right)$ is the integrated excitation intensity of the incident beam illuminating the sample (an empty sphere). The denominator of Eq. 8 represents the total number of photons absorbed by the sample.

Two sources of uncertainty exist in Eq. 8. The first involves the spectral responsivity of the integrating sphere/detection system. The second stems from potential sample reabsorption effects resulting from the large effective optical path length introduced by the integrating sphere. The former can be addressed by correcting the overall spectral responsivity of the system to the known spectral radiance of a reference light source. For the latter, emission reabsorption can be accounted for by correcting measured QYs using ${ }^{90}$

$$
\mathrm{QY}_{\text {absolute }}=\frac{\mathrm{QY}_{\text {observed }}}{1-\mathrm{r}\left(1-\mathrm{QY}_{\text {Observed }}\right)},
$$

where $r$ is the reabsorption probability, estimated from the ratio of integrated emission intensities of concentrated and dilute $\mathrm{NC} /$ nanostructure solutions ${ }^{82}$.

\section{Power-dependent photoluminescence}

An alternative approach to measuring absolute QYs is called PDPL. PDPL involves measuring $I_{\mathrm{em}}$ as a function of $I_{\text {exc }}$. The resulting $I_{\text {em }}$ versus $I_{\text {exc }}$ dependency exhibits a peaked structure that can be fit with a kinetic model to yield absolute QYs. These optimal, excitation intensitydependent QYs are crucial parameters for establishing the likelihood of laser cooling. Peaked QY $I_{\text {exc }}$ dependencies have previously been illustrated in Fig. 5c and Fig. 6b. Whereas both pulsed ${ }^{57,91,92}$ and continuous wave $(\mathrm{CW})^{93}$ laser sources have been employed for PDPL, the following model description assumes the use of a CW laser. Specifics regarding the pulsed case modeling can be found in refs. ${ }^{57,91,92}$.

A generic model for carrier recombination in a semiconductor is first constructed by consolidating Eqs. 1 and 2 into

$$
\frac{\mathrm{d} N(t)}{\mathrm{d} t}=\frac{\alpha\left(\nu_{\mathrm{exc}}\right) I_{\mathrm{exc}}}{h \nu_{\mathrm{exc}}}-\left[A N(t)+\eta_{\mathrm{e}} B N(t)^{2}+C N(t)^{3}\right]
$$

under the assumption of equal photogenerated electron and hole carrier densities. In Eq. 10, $N$ is the free charge carrier density, $v_{\text {exc }}$ is the excitation frequency, $\alpha\left(v_{\text {exc }}\right)$ is the $\mathrm{NC} /$ nanostructure absorption coefficient at $v_{\mathrm{exc}}, \eta_{\mathrm{e}}$ is a photon extraction efficiency, and $A, B$, and $C$ are the semiconductor's rate constants, associated with first-order carrier trapping, second-order radiative recombination, and third-order Auger nonradiative recombination.

At steady state, $\frac{\mathrm{d} N(t)}{\mathrm{d} t}=0$; thus, $I_{\text {exc }}$ can alternatively be expressed in terms of the steady-state carrier density, $N$, as

$$
I_{\mathrm{exc}}=\frac{h \nu_{\mathrm{exc}}}{\alpha\left(\nu_{\mathrm{exc}}\right)}\left[A N+\eta_{\mathrm{e}} B N^{2}+C N^{3}\right] .
$$

A corresponding emission intensity is

$$
I_{\mathrm{em}}=\kappa h \nu_{\mathrm{em}} \eta_{\mathrm{e}} B N^{2},
$$

where $\kappa$ is a proportionality constant with units of length that depends upon instrument geometry, sample thickness, and detector collection efficiency. The ratio of $I_{\mathrm{em}}$ to $I_{\text {exc }}$ then gives

$$
\frac{I_{\mathrm{em}}}{I_{\mathrm{exc}}}=\frac{\kappa \nu_{\mathrm{em}} \alpha\left(\nu_{\mathrm{exc}}\right)}{\nu_{\mathrm{exc}}} \mathrm{QY}(N)
$$

where

$$
\mathrm{QY}(N)=\frac{\eta_{\mathrm{e}} B N^{2}}{A N+\eta_{\mathrm{e}} B N^{2}+C N^{3}} .
$$

In practice, $\mathrm{QY}(N)$ exhibits a peaked functional form with $I_{\text {exc }}$ due to the various competing carrier recombination processes that underlie it. This has previously been illustrated in Figs. $5 \mathrm{c}$ and $6 \mathrm{~b}$, where, at low excitation intensities, the QY grows with $I_{\text {exc }}$, being limited by nonradiative carrier trapping processes. At higher intensities, the QY peaks due to trap saturation. At even higher intensities, the QY decreases with increasing $I_{\text {exc }}$ due to the onset of nonradiative Auger recombination.

By taking the derivative of Eq. 14 with respect to $N$, the carrier density associated with the maximum observable QY is

$$
N_{\mathrm{opt}}=\sqrt{\frac{A}{C}}
$$


and is linked with a corresponding optimal excitation intensity of

$$
I_{\mathrm{opt}} \approx \frac{h \nu_{\mathrm{exc}}}{\alpha\left(\nu_{\mathrm{exc}}\right)} \eta_{\mathrm{e}} B N_{\mathrm{opt}}^{2}=\frac{h \nu_{\mathrm{exc}}}{\alpha\left(\nu_{\mathrm{exc}}\right)} \eta_{\mathrm{e}} \frac{A B}{C} .
$$

$I_{\text {opt }}$ is found from Eqs. 13 and 14 when $B N^{2} \gg A N+$ $C N^{3}$. The sample's peak QY, in terms of $N_{\text {opt }}$, is then

$$
\mathrm{QY}_{\mathrm{opt}}=\frac{\eta_{\mathrm{e}} B N_{\mathrm{opt}}^{2}}{A N_{\mathrm{opt}}+\eta_{\mathrm{e}} B N_{\mathrm{opt}}^{2}+C N_{\mathrm{opt}}^{3}}=\frac{1}{1+\left(\frac{2 \sqrt{A C}}{\eta_{\mathrm{e}} B}\right)} \approx 1-\frac{2 \sqrt{A C}}{\eta_{\mathrm{e}} B} .
$$

In the ideal case, the rate constants $A, B$, and $C$ are known. The photon extraction efficiency, $\eta_{\mathrm{e}}$, is furthermore measurable ${ }^{94}$. Consequently, Eq. 17 immediately yields the maximum absolute QY of a sample.

In practice, however, $\mathrm{NC} /$ nanostructure rate constants are often not well characterized and, for NCs, can be sizedependent ${ }^{95,96}$. The practical employment of PDPL thus requires additional steps to be taken. To proceed, a dimensionless excitation intensity, $\bar{I}_{\text {exc }}$, is therefore defined by normalizing $I_{\text {exc }}$ to $I_{\text {opt }}$. What results is

$$
\bar{I}_{\mathrm{exc}}=\frac{I_{\mathrm{exc}}}{I_{\mathrm{opt}}} \approx \frac{N^{2}}{N_{\mathrm{opt}}^{2}} .
$$

Assuming the limit of near-unity QYs and replacing $N$ in Eq. 14 with $N=N_{\text {opt }} \sqrt{\bar{I}_{\text {exc }}}$ (Eq. 18) yields the following QY expression:

$$
\mathrm{QY}\left(\bar{I}_{\mathrm{exc}}\right)=1-\frac{A \bar{I}_{\mathrm{exc}}^{\frac{1}{2}} N_{\mathrm{opt}}+C \bar{I}_{\mathrm{exc}}^{\frac{3}{2}} N_{\mathrm{opt}}^{3}}{\eta_{\mathrm{e}} B \bar{I}_{\mathrm{exc}} N_{\mathrm{opt}}^{2}} .
$$

Then, using $N_{\text {opt }}$ from Eq. 15 gives

$$
\mathrm{QY}\left(\bar{I}_{\mathrm{exc}}\right)=1-\frac{2 \sqrt{A C}}{\eta_{\mathrm{e}} B} \frac{1+\bar{I}_{\mathrm{exc}}}{2 I_{\mathrm{exc}}^{\frac{1}{2}}} \text {. }
$$

In terms of $\mathrm{QY}_{\mathrm{opt}}$ (Eq. 17), this is

$$
\mathrm{QY}\left(\bar{I}_{\mathrm{exc}}\right)=1-\left(1-\mathrm{QY}_{\mathrm{opt}}\right) \frac{1+\bar{I}_{\mathrm{exc}}}{2 \bar{I}_{\mathrm{exc}}^{\frac{1}{2}}} \text {. }
$$

Finally, introducing $\mathrm{QY}_{\mathrm{opt}}\left(\bar{I}_{\text {exc }}\right)$ into the ratio $\frac{I_{\text {em }}}{I_{\text {exc }}}$ (Eq. 13) gives

$$
\frac{I_{\mathrm{em}}}{I_{\mathrm{exc}}}=\frac{\kappa \nu_{\mathrm{em}} \alpha\left(\nu_{\mathrm{exc}}\right)}{\nu_{\mathrm{exc}}}\left[1-\left(1-\mathrm{QY}_{\mathrm{opt}}\right) \frac{1+\bar{I}_{\mathrm{exc}}}{2 \bar{I}_{\mathrm{exc}}^{\frac{1}{2}}}\right] \text {. }
$$

Experimental $\frac{I_{\text {em }}}{I_{\text {exc }}}$ versus $I_{\text {exc }}$ data can therefore be fit with Eq. 22 to find a NC's/nanostructure's $\mathrm{QY}_{\mathrm{opt}}$. This fitting procedure has previously been illustrated in Fig. $6 \mathrm{~b}$ for a $\mathrm{MAPbI}_{3}$ single crystal. In general, the approach is relatively fast and does not require any special corrections for detector responsivity. PDPL, however, yields accurate QYs only when they are near-unity in value given approximations made in the modeling. Additional details of the technique can be found in ref. ${ }^{93}$.

\section{Calorimetric approaches}

A third class of QY measurements entails calorimetric approaches. These measurements estimate $\mathrm{NC}$ /nanostructure QYs based on local heating that arises from nonradiative relaxation in the sample following excitation. Of this class of techniques, popular examples include thermal lensing and photoacoustic spectroscopy ${ }^{97-99}$. Neither, however, is commonly used to estimate $\mathrm{NC} /$ nanostructure QYs due to difficulties in achieving accurate values. This stems, in part, from the fact that these techniques work best on lower-QY samples, where a sizable fraction of the excitation causes local heating.

\section{All optical scanning laser calorimetry}

An alternative approach that circumvents these issues is called all optical scanning laser calorimetry (ASLC), which measures QYs through a sample's temperature-dependent emission spectrum ${ }^{91,100}$. To illustrate how ASLC links a sample's peak emission frequency to its QY, we first describe its heating power, $P_{\text {heat }}$ due to nonradiative recombination, following excitation at a frequency, $v_{\mathrm{exc}}$. Ignoring parasitic/ background absorption, $P_{\text {heat }}$ can be expressed as the difference between absorbed and emitted powers:

$$
\begin{gathered}
P_{\text {heat }}=\alpha\left(\nu_{\mathrm{exc}}\right) I_{\mathrm{exc}}-h \nu_{\mathrm{em}} \eta_{\mathrm{e}} B N^{2} \\
=\eta_{\mathrm{e}} B N^{2}\left(h \nu_{\mathrm{exc}}-h \nu_{\mathrm{em}}\right)+A N h \nu_{\mathrm{exc}}+C N^{3} h \nu_{\mathrm{exc}} \\
=P_{\mathrm{em}}\left(\frac{1}{\mathrm{QY}} \frac{\nu_{\mathrm{ex}}}{\nu_{\mathrm{em}}}-1\right),
\end{gathered}
$$

where $v_{\mathrm{em}}$ is the mean emission frequency and $P_{\mathrm{em}}=$ $h v_{\mathrm{em}} \eta_{\mathrm{e}} B N^{2}$ is the corresponding emission power. If $P_{\text {heat }}$ proportionally induces a temperature change, $\Delta T$, Eq. 23 can be rewritten as

$$
\Delta T \propto P_{\mathrm{em}}\left(\frac{1}{\mathrm{QY}} \frac{\nu_{\mathrm{exc}}}{\nu_{\mathrm{em}}}-1\right) .
$$

In practice, a specimen's temperature-dependent emission spectrum is acquired to generate a $\Delta T$ calibration curve. Then, a separate measurement varies $v_{\text {exc }}$ while keeping $P_{\text {em }}$ constant. At each frequency, $\Delta T$ is estimated and plotted against $v_{\text {exc }}$. Equation 24 shows that a linear relationship exists between the two parameters such that the acquired experimental data can be extrapolated to the intercept where $\Delta T=0$. This is illustrated conceptually in Fig. 7. The sample's emission QY is then found using the ratio

$$
\mathrm{QY}=\frac{\nu_{\mathrm{exc}, \Delta \mathrm{T}=0}}{\nu_{\mathrm{em}}} .
$$

This illustrates the convenience of ASLC and demonstrates its suitability to samples not readily amenable to 


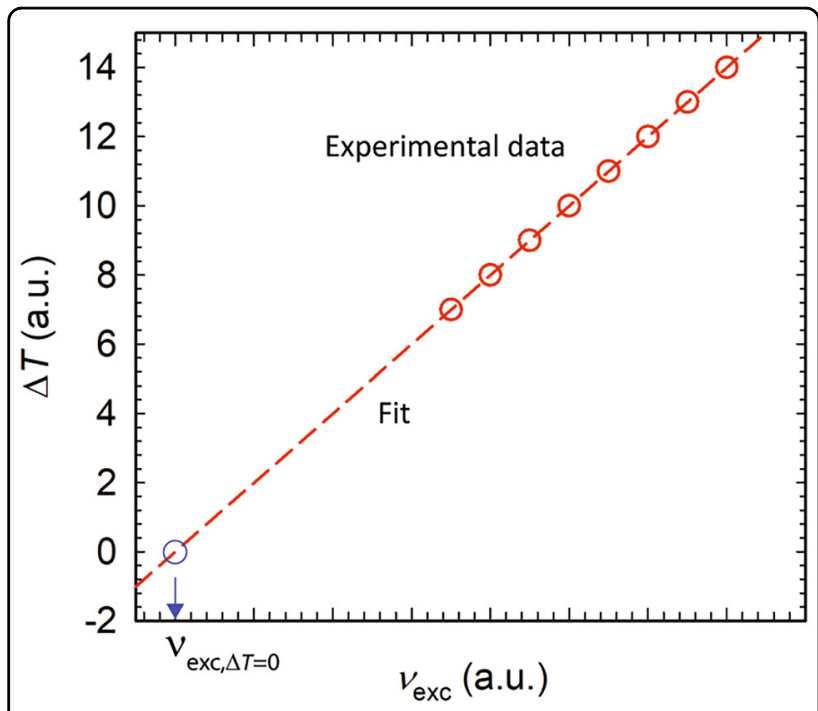

Fig. 7 Schematic representation of temperature-dependent data obtained by ASLC

relative or absolute integrating sphere approaches. Of note, ASLC is applicable to conducting single $\mathrm{NC} /$ nanostructure QY measurements ${ }^{50}$.

In all cases, accurate ASLC QYs require accounting for the spectral responsivity of the detection system as well as maintaining a high degree of sample temperature stability. More importantly, specimens should exhibit clear temperature-dependent changes to $v_{\mathrm{em}}$. In this regard, systems such as $\mathrm{CsPbBr}{ }_{3} \mathrm{NCs}$ exhibit small $\Delta T$ variations of their emission spectra ${ }^{101}$, making ASLC measurements problematic. Finally, Figs. $5 \mathrm{c}$ and $6 \mathrm{~b}$ show that QYs depend upon $I_{\text {exc }}$. Consequently, ASLC should be conducted at $I_{\text {opt }}$ to establish a sample's maximum QY. In practice, $I_{\mathrm{opt}}$ is found using PDPL, carried out in conjunction with ASLC.

Table 2 summarizes the experimentally reported maximum QYs of popular semiconductor $\mathrm{NC}$ /nanostructure systems established using the above-mentioned relative, absolute, and calorimetric approaches.

\section{Estimating upconversion efficiencies}

Beyond QY, Eq. 5 shows that equally important to achieving condensed phase laser cooling are experimental $\mathrm{NC} /$ nanostructure $\eta_{\mathrm{ASPL}}$ values. Notably, although $\mathrm{NC} /$ nanostructure emission upconversion has been reported for many years ${ }^{53,59-61,63-65,72,102}$, few studies have actually estimated $\eta_{\mathrm{ASPL}}$. Those that have report values between $3.34 \times 10^{-4}$ and $2.7 \times 10^{-2}$.

In practice, experimental $\eta_{\text {ASPL }}$ values can be established using

$$
\eta_{\mathrm{ASPL}}=\frac{I_{\mathrm{exc}, \text { Stokes }} A_{\text {Stokes }}}{I_{\mathrm{exc}, \mathrm{ASPL}} A_{\mathrm{anti}-\text { Stokes }}},
$$

Table 2 Experimental maximum NC/nanostructure QYs

\begin{tabular}{|c|c|c|c|}
\hline System & $\mathrm{QY}_{\max }(\%)$ & Measurement technique & Ref. \\
\hline CdSe NC & 100 & Absolute & 131 \\
\hline \multirow[t]{4}{*}{$\mathrm{CdSe} / \mathrm{CdS} \mathrm{NC}$} & $90-98$ & Absolute & 132 \\
\hline & 94 & Absolute & 133 \\
\hline & $94-97$ & NA & 134 \\
\hline & 100 & Absolute & 135 \\
\hline $\mathrm{CdSe} / \mathrm{CdS} \mathrm{NR}$ & $95-98$ & Absolute & 136 \\
\hline \multirow[t]{3}{*}{$\mathrm{CsPbBr}_{3} \mathrm{NC}$} & $\sim 100$ & Absolute & 137 \\
\hline & $\sim 100$ & Relative & 73 \\
\hline & 93 & Absolute & 138 \\
\hline \multirow[t]{2}{*}{$\mathrm{CsPb}_{3} \mathrm{NC}$} & $\sim 100$ & Absolute & 139 \\
\hline & 95 & Absolute & 138 \\
\hline $\mathrm{MAPbl}_{3}$ & 99.8 & ASLC & 50 \\
\hline $\mathrm{MAPbBr}_{3} \mathrm{NC}$ & 100 & Absolute & 140 \\
\hline $\mathrm{MoS}_{2}$ monolayer & $>95$ & Custom & 141 \\
\hline
\end{tabular}

where $I_{\text {exc,Stokes }}$ and $I_{\text {exc,ASPL }}$ are the Stokes and antiStokes excitation intensities required to achieve identical Stokes/ASPL emission intensities (i.e., $I_{\mathrm{em}}=I_{\mathrm{ASPL}}$ ). $A_{\text {Stokes }}$ and $A_{\text {anti-Stokes }}$ are the corresponding Stokes and antiStokes absorptance values found using $A=1-10^{-\mathrm{Abs}}$, where Abs is the absorbance of the $\mathrm{NC} /$ nanostructure at $I_{\text {exc,Stokes }}$ or $I_{\text {exc,ASPL }}$. Equation 26 implicitly assumes the involvement of real intermediate states in the upconversion process.

Using Eq. 26, we find that $\mathrm{CsPbBr}_{3}$ and $\mathrm{CdSe} / \mathrm{CdS} \mathrm{NCs}$ possess sizable $\eta_{\text {ASPL }}$ values. Specifically, for $\mathrm{CsPbBr}_{3}$ $\mathrm{NCs}, \eta_{\mathrm{ASPL}}=0.75\left(\eta_{\mathrm{ASPL}}=0.32\right)$ for $\Delta E=23(\Delta E=102)$ $\mathrm{meV}^{103}$. For $\mathrm{CdSe} / \mathrm{CdS}$ core/shell $\mathrm{NCs}, \eta_{\mathrm{ASPL}}=0.55$ $\left(\eta_{\mathrm{ASPL}}=0.03\right)$ for $\Delta E=56.6(\Delta E=107)$. These and all other reported $\eta_{\text {ASPL }}$ values are tabulated below in Table 3.

\section{Experimental $\mathrm{NC} /$ nanostructure $\mathrm{QYs}$ and $\eta_{\mathrm{ASPL}}$ values}

Table 3 now summarizes all experimental NC/nanostructure QY and corresponding $\eta_{\text {ASPL }}$ values we are aware of in the literature. Upon inspection, it is evident that many $\mathrm{NC} /$ nanostructure systems exhibit ASPL. It is also clear that much of the older literature addresses samples with suboptimal QYs. Newer systems such as CdS, CdSe, and hybrid/all inorganic lead halide perovskites [e.g., $\mathrm{MAPbI}_{3}$ and cesium lead bromide $\left.\left.(\mathrm{CsPbBr})_{3}\right)\right]$ show much more promising QY and $\eta_{\text {ASPL }}$ values. These systems should therefore be focused on in future NC-based laser cooling studies. Other NCs/nanostructures, which today 
Table 3 Summary of experimental ASPL instances in NCs/nanostructures

\begin{tabular}{|c|c|c|c|c|}
\hline System & QY (\%) & $\eta_{\text {ASPL }}$ & $\langle\Delta E\rangle, \Delta E_{\max }(\mathrm{meV})$ & Ref. \\
\hline $\mathrm{TiO}_{2} \mathrm{NCS}$ & 0.25 & - & $\langle\Delta E\rangle=270-1120$ & 53 \\
\hline \multirow[t]{2}{*}{ CdS NBs } & $10-64$ & 0.1 & $\langle\Delta E\rangle=124-860$ & 57 \\
\hline & $77-99$ & - & $\langle\Delta E\rangle=100$ & 107 \\
\hline \multirow[t]{3}{*}{ CdSe/ZnS NCs } & 55 & $2.7 \times 10^{-2}$ & $\Delta E_{\max }=335$ & 59 \\
\hline & $>50$ & $1.7 \times 10^{-3}$ & $a x=413$ & 61 \\
\hline & - & $1.7 \times 10^{-3}$ & $\Delta E_{\max }=413$ & 60 \\
\hline $\mathrm{CdSe} / \mathrm{CdS} \mathrm{NCs}$ & 73 & $0.55(0.03)$ & $\langle\Delta E\rangle=57(\langle\Delta E\rangle=107)$ & This study \\
\hline \multirow[t]{4}{*}{ CdTe NCs } & 15 & $1.35 \times 10^{-2}$ & $\Delta E_{\max }=285$ & 59 \\
\hline & 15 & $8.7 \times 10^{-3}-1.3 \times 10^{-2}$ & $\Delta E_{\max }=279-319$ & 63 \\
\hline & - & - & $\Delta E_{\max }=360$ & 65 \\
\hline & 20 & $3.34 \times 10^{-4}$ & $\Delta E_{\max }=290-350$ & 64 \\
\hline \multirow[t]{3}{*}{ PbS NCs } & - & - & $\langle\Delta E\rangle \sim 10$ & 102 \\
\hline & - & - & $\Delta E_{\max }=330$ & 72 \\
\hline & - & - & $\langle\Delta E\rangle=18-60, \Delta E_{\max }=240-430$ & 67 \\
\hline $\mathrm{MAPbl}_{3}$ & 99.8 & - & $\langle\Delta E\rangle=27$ & 50 \\
\hline $\mathrm{PhEPbl}_{4}$ & - & - & $\langle\Delta E\rangle=386$ & 50 \\
\hline \multirow[t]{2}{*}{$\mathrm{CsPbBr}_{3} \mathrm{NCs}$} & $\sim 85$ & $0.75(0.32)$ & $\langle\Delta E\rangle=23(\langle\Delta E\rangle=102)$ & 103 \\
\hline & $68-100$ & $0.11-0.33$ & $\langle\Delta E\rangle=64-78$ & 73 \\
\hline $\mathrm{CsPb}\left(\mathrm{Br} / \mathrm{l}_{3} \mathrm{NCs}\right.$ & - & - & $\langle\Delta E\rangle=9.3-191, \Delta E_{\max }=430$ & 106 \\
\hline
\end{tabular}

show high QYs, will likely upconvert and should also be investigated.

\section{Towards a mechanistic understanding of NC/ nanostructure $I_{\text {ASPL }}$}

We now summarize what is understood about $\mathrm{NC} /$ nanostructure ASPL using the above-compiled literature observations. To begin, it generally involves real intermediate states related to defects. This is because excitation intensities in many experiments are often low and are below the values expected for two-photon processes. Consequently, only real intermediate states with finite lifetimes are expected to yield sizable ASPL intensities.

Next, NC/nanostructure upconversion is most often a one-photon process relevant to laser cooling. This conclusion is supported by the observation of linear $I_{\mathrm{ASPL}}$ and $I_{\text {exc }}$ dependencies in NC systems, such as $\mathrm{TiO}_{2}{ }^{53}, \mathrm{CdS}^{56}$, $\mathrm{CdSe}^{58-62,104}, \mathrm{CdTe}^{59,63-65,105}, \mathrm{PbS}^{67}, \mathrm{CsPbBr}_{3}{ }^{73,103}$, and $\mathrm{CsPbBrI}_{2}{ }^{69,106}$. Other nanostructures exhibiting linear $I_{\mathrm{ASPL}}$ versus $I_{\text {exc }}$ behavior include $\mathrm{CdS} \mathrm{NBs}^{47,57,107}, \mathrm{ZnTe}$ nanoribbons ${ }^{108}$, bulk $\mathrm{MAPbI}_{3}{ }^{50}$, and $\mathrm{PhEPbI}_{3}$ nanoplatelets ${ }^{50}$. Although occasional quadratic/near-quadratic growth has been reported ${ }^{68,70,71,109-113}$, the bulk of the available literature points to the dominant role played by one-photon and, by corollary, defect-mediated processes in $\mathrm{NC} /$ nanostructure ASPL.

Phonon involvement is confirmed by reported ASPL temperature $(T)$ dependencies. Namely, $I_{\text {ASPL }}$ increases (decreases) with increasing (decreasing) temperature. This has been reported for $\mathrm{CdS}^{57}, \mathrm{CdSe}^{61,62}, \mathrm{CdTe}^{59,63-65}$, and $\mathrm{CsPbBr}_{3} \mathrm{NCs}^{73}$, as well as for ZnTe nanoribbons ${ }^{108}$. These positive dependencies, in turn, are consistent with the Boltzmann population of phonon states. As a point of contrast, $\mathrm{NC} /$ nanostructure $I_{\mathrm{em}}$ values generally scale inversely with temperature.

Less frequently reported but equally relevant is the observation that $I_{\mathrm{ASPL}}$ increases (decreases) exponentially with decreasing (increasing) detuning (i.e., $\Delta E$ ) of the excitation laser into the semiconductor gap. This has been reported for $\mathrm{CdS}^{57}$ and $\mathrm{CsPbBrI}_{2} \mathrm{NCs}^{69}$, as well as for free-standing $\mathrm{GaN}$ films ${ }^{114}$. These dependencies again point to phonon involvement given the exponential $\Delta E$ dependency of the Boltzmann distribution.

The qualitative picture that emerges is ASPL from defect-mediated, one-photon/phonon upconversion to the semiconductor band edge. A schematic of the process is illustrated in Fig. 2b. This conclusion is further 
supported by associated kinetic modeling using

$$
\begin{aligned}
& \frac{\mathrm{d} n}{\mathrm{~d} t}=k_{\mathrm{ph}} n_{\mathrm{t}}-k_{\mathrm{r}} n p-k_{\mathrm{t}} n\left(N_{\mathrm{t}}-n_{\mathrm{t}}\right)-k_{\text {Auger }} n p^{2}, \\
& \frac{\mathrm{d} p}{\mathrm{~d} t}=G^{\prime}-k_{\mathrm{r}} n p-k_{\mathrm{p}} n_{\mathrm{t}} p-k_{\text {Auger }} n p^{2}, \\
& \frac{\mathrm{d} n_{t}}{\mathrm{~d} t}=G^{\prime}+k_{\mathrm{t}} n\left(N_{\mathrm{t}}-n_{\mathrm{t}}\right)-k_{\mathrm{ph}} n_{\mathrm{t}}-k_{\mathrm{p}} n_{\mathrm{t}} p,
\end{aligned}
$$

where $G^{\prime}$ is the absorbed subgap excitation rate $\left(G^{\prime} \propto\right.$ $I_{\text {exc,ASPL }}$ ).

At low excitation intensities, $I_{\mathrm{ASPL}}$ takes the limiting form

$$
I_{\mathrm{ASPL}}=\left(\frac{k_{\mathrm{r}} k_{\mathrm{ph}}^{\mathrm{o}}}{k_{\mathrm{t}} k_{\mathrm{p}}}\right)\left(\frac{G^{\prime}}{N_{\mathrm{t}}}\right) e^{-\frac{\Delta E}{k T}}
$$

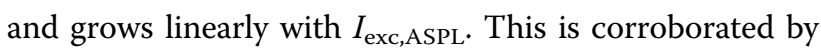
numerical solutions to Eqs. 27-29 using parameters previously used to simulate Eqs. 1-3. The only difference is the use of a larger $k_{\text {ph }}$ value (i.e., $k_{\mathrm{ph}}^{\mathrm{o}}=10^{8} \mathrm{~s}^{-1}$ ) to model efficient upconversion. Figure 8 shows plots of $I_{\mathrm{ASPL}}$ for different $N_{\mathrm{t}}$ values, wherein $I_{\mathrm{ASPL}}$ follows the predicted linear dependence at low excitation intensities.

Equation 30 additionally reveals that $I_{\mathrm{ASPL}}$ increases (decreases) with increasing temperature (increasing $\Delta E$ ). Specifically, $\ln \left(I_{\mathrm{ASPL}}\right) \propto-\left(\frac{\Delta E}{k}\right) \frac{1}{T}=-\left(\frac{1}{k T}\right) \Delta E$. These predictions agree with the above-mentioned experimental observations.

Notably, Eq. 30 predicts that $I_{\text {ASPL }}$ possesses an inverse $N_{\mathrm{t}}$ dependence. Lowering $\mathrm{NC} /$ nanostructure trap densities should therefore increase $I_{\mathrm{ASPL}}$. This last prediction is important because it has been observed that $I_{\mathrm{ASPL}}$

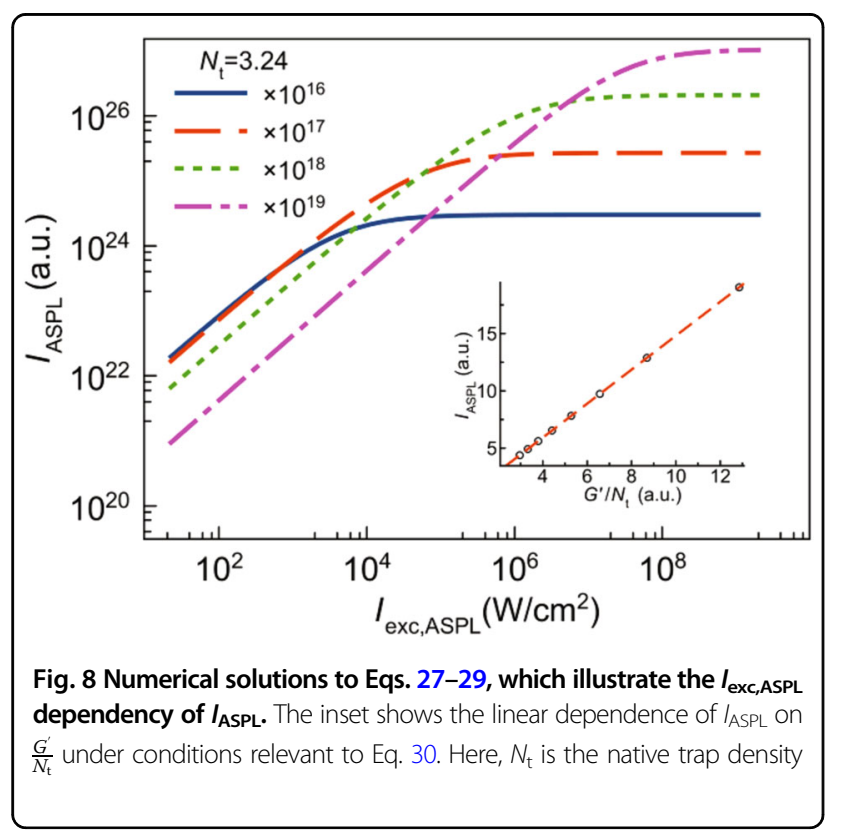

increases with increasing QYs in both $\mathrm{CdSe}^{60,61}$ and $\mathrm{CsPbBr}_{3} \mathrm{NCs}^{73}$. We also observe this behavior in CdSe/ CdS NCs studied here.

This positive $I_{\mathrm{ASPL}} / \mathrm{QY}$ correlation was initially interpreted as a sign that ASPL may not be defect-mediated, as $N_{\mathrm{t}}$ is directly associated with $\mathrm{NC} /$ nanostructure QYs. Minimizing defect-state densities might therefore be expected to reduce upconversion activity. The kinetic model, however, rationalizes this observation and reveals that increased QYs are, in fact, consistent with enhanced $I_{\mathrm{ASPL}}$ values.

This observation is explicitly illustrated in the inset of Fig. 8, where $I_{\mathrm{ASPL}}$ has been plotted as a function of $\frac{G^{\prime}}{N_{\mathrm{t}}}$ using numerical solutions to Eqs. 27-29. A clear linear dependence is seen, as first predicted by Eq. 30 . The linearity spans the range of trap densities $N_{\mathrm{t}}=10^{20}-10^{21}$ $\mathrm{cm}^{-3}$, where the condition $N_{\mathrm{t}} \gg n_{\mathrm{t}}$ holds.

Finally, at high excitation intensities and low trap state densities (e.g., $N_{\mathrm{t}}=3.24 \times 10^{16} \mathrm{~cm}^{-3}$ ), trap saturation occurs such that $n_{\mathrm{t}} \approx N_{\mathrm{t}}$. Under these conditions, it can be shown analytically that

$$
I_{\mathrm{ASPL}} \approx k_{\mathrm{ph}} N_{\mathrm{t}}
$$

$I_{\mathrm{ASPL}}$ saturates and is corroborated by the numerical results in Fig. 8. Similar saturation behavior is predicted at other $N_{\mathrm{t}}$ values.

There is one final observation to explain. This entails observations of redshifted ASPL spectra relative to the normal $\mathrm{NC} /$ nanostructure band edge emission. These redshifts have been observed in $\mathrm{CdS}_{1-x} \mathrm{Se}_{x}{ }^{110}$, $\mathrm{CdS}^{59,62,109}, \mathrm{CdTe}^{59,63,65,66,71,105,112,113}, \mathrm{PbS}$ NC-doped glasses $^{67,115}, \mathrm{CsPbBr}_{3}{ }^{73,103}$, and $\mathrm{CsPbBrI}_{2} \mathrm{NCs}^{69}$. Figure 9a provides an example, showing the apparent $\sim 8 \mathrm{meV}$ redshift between the emission and ASPL spectra of a CsPbBr $\mathrm{NC}$ ensemble. These redshifts have, in turn, been used to suggest that ASPL originates from different $\mathrm{NC}$ /nanostructure (defect) states, unrelated to the normal, above gap-excited, NC/nanostructure emitting state ${ }^{62,65,67}$.

We suggest that these redshifts are artifacts related to $\mathrm{NC} /$ nanostructure ensemble residual size distributions. This is because apparent redshifts have not been observed in the ASPL spectra of individual $\mathrm{CsPbBr}_{3} \mathrm{NCs}^{103}$ and $\mathrm{CdS} \mathrm{NBs}^{57}$. Figure 9b, c provide illustrations, revealing that single $\mathrm{CsPbBr}_{3} \mathrm{NC}^{103}$ and $\mathrm{CdS} \mathrm{NB}^{57}$ emission and ASPL spectra exhibit near-coincident energies.

Additionally, an ASPL redshift arises naturally when accounting for an ensemble's residual size distribution. In particular, convoluting a single NC's thermally broadened emission spectrum with a modified Gaussian function that accounts for the ensemble size distribution, the Boltzmann upconversion probability in Eq. 30, and the 


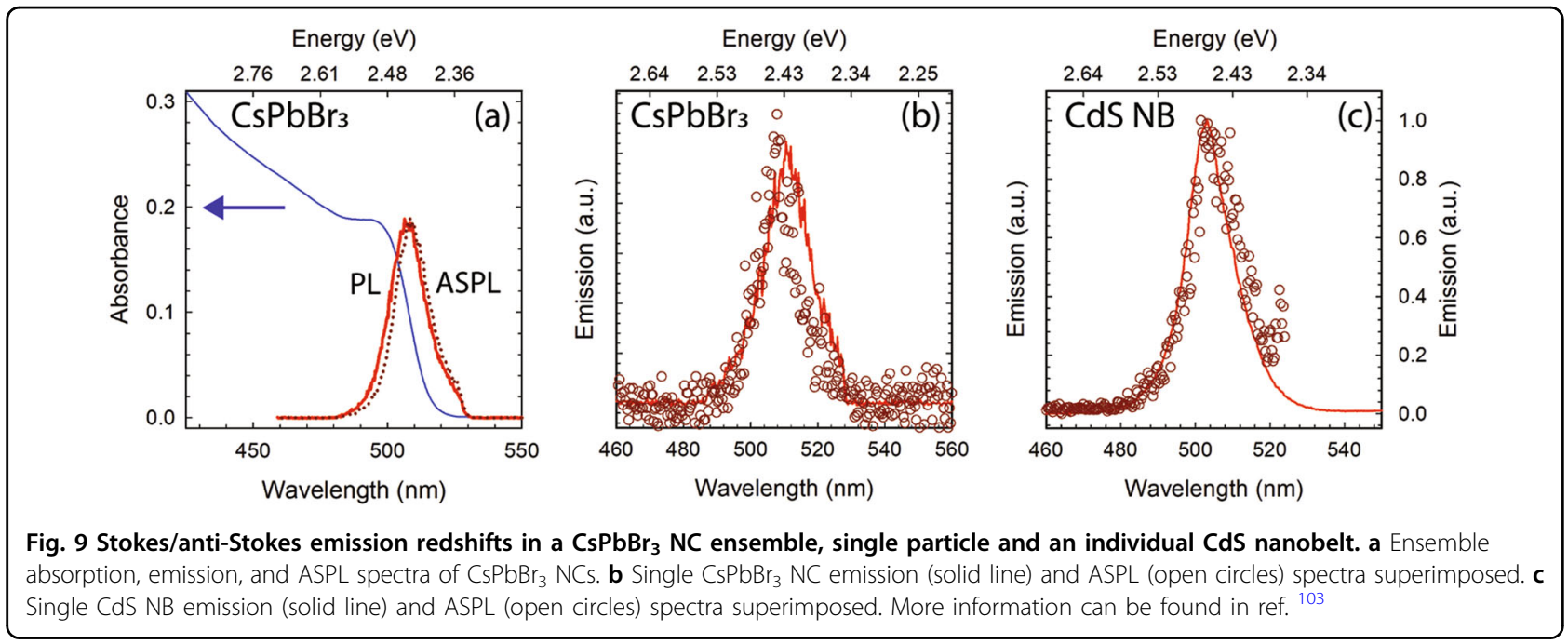

exponential decrease in absorption into the gap [Abs $(x)]$

$$
\begin{gathered}
I_{\mathrm{ASPL}}(E)=\int_{0}^{\infty} \operatorname{Abs}(\mathrm{x}) \mathrm{e}^{-\frac{E^{\prime}(x)-E_{\mathrm{exc}}}{\mathrm{kT}}}\left[\frac{1}{\sqrt{2 \pi \sigma_{x}^{2}}} e^{\frac{-(x-\bar{x})^{2}}{2 \sigma_{x}^{2}}}\right] \\
{\left[\frac{1}{\sqrt{2 \pi \sigma_{E}^{2}}} e^{\frac{-\left(E-E^{\prime}(x)\right)^{2}}{2 \sigma_{E}^{2}}}\right] \mathrm{d} x}
\end{gathered}
$$

yields an $I_{\mathrm{ASPL}}$ spectrum naturally redshifted from the normal emission spectrum. In Eq. 32, $\sigma_{x}$ reflects the ensemble's size distribution, and $\bar{x}$ is the mean $\mathrm{NC} /$ nanostructure size. $\sigma_{E}$ accounts for the thermal broadening of a single NC's/nanostructure's emission linewidth, with $E^{\prime}(x)$ representing the mean emission energy for a given size and $E_{\text {exc }}$ denoting the excitation energy.

By plotting $I_{\mathrm{ASPL}}(E)$, it is evident that a redshift of the ASPL spectrum appears naturally. This is illustrated in Fig. 10, where the ASPL redshift due to a $\mathrm{CsPbBr}_{3} \mathrm{NC}$ ensemble's size distribution has been simulated. Employed parameters include $\bar{x}=9.2 \mathrm{~nm}, \sigma_{x}=0.5$ $-1.5 \mathrm{~nm}$, and $\sigma_{E}=0.04 \mathrm{eV}$. $\operatorname{Abs}(x)$ is found by fitting the red edge of the experimental NC absorption spectrum, while $E^{\prime}(x)$ is found using a sizing curve from the literature, linking emission maxima to $\mathrm{NC}$ size ${ }^{79}$.

\section{$\mathrm{CsPbr}_{3} \mathrm{NC}$ cooling simulation}

A global survey of Tables 1, 2, and 3 identifies $\mathrm{CsPbBr}_{3}$ $\mathrm{NCs}$ as a particularly promising material for laser cooling demonstrations. This stems from critical QYs as low as $\mathrm{QY}_{\text {crit }}=96 \%$, experimental near-unity QYs, and sizable $\eta_{\text {ASPL }}$ values. To investigate the potential cooling performance of this material, we numerically simulated its cooling when dispersed within a thermally isolating aerogel matrix. The simulations consider photogenerated charge carrier recombination, include Auger recombination and model heat diffusion across the NC composite.

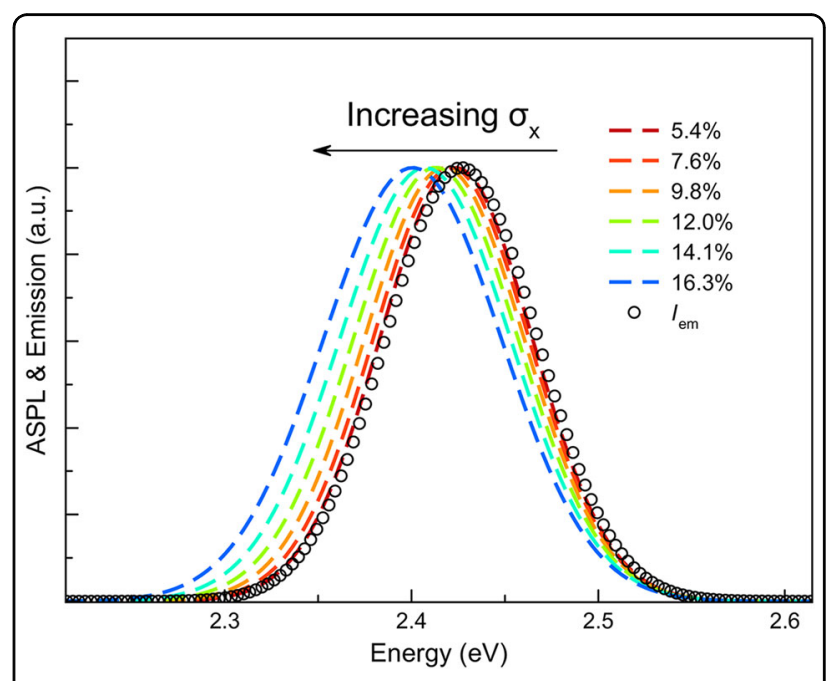

Fig. 10 Simulated ASPL redshift of a $\mathrm{CsPBBr}_{3} \mathrm{NC}$ ensemble relative to its normal above gap-excited emission

Figure 11a illustrates the NC solid, where $\mathrm{CsPbBr}_{3} \mathrm{NCs}$ have been uniformly dispersed within the inner portion of a 20-mm-diameter aerogel disk. The inner diameter is $2 \mathrm{~mm}$, and the overall aerogel thickness is $0.1 \mathrm{~mm}$. An associated NC density in the inner disk is $3.1 \times 10^{16} \mathrm{~cm}^{-3}$. The aerogel is thermally clamped to a $T_{\mathrm{o}}=300 \mathrm{~K}$ reservoir around its perimeter, and NCs are excited below-gap using a $532 \mathrm{~nm}$ laser with an intensity resulting in an effective carrier generation rate of $G^{\prime}=1.22 \times 10^{22} \mathrm{~cm}^{3}$ $\mathrm{s}^{-1}$. Due to the relatively small absorption cross-section for below-gap excitation, the incident excitation is assumed to occur homogeneously throughout the inner disk containing NCs.

The kinetic expressions for charge generation and recombination are analogous to those shown in Eqs. 2729 with one difference. Given reports of unity QYs (Table 
2), $n_{\mathrm{t}} \ll n$. What results is

$$
\frac{\mathrm{d} n}{\mathrm{~d} t} \approx \frac{\mathrm{d} p}{\mathrm{~d} t} \approx G^{\prime}-k_{\mathrm{r}} n p-k_{\text {Auger }} n p^{2},
$$

with $k_{\mathrm{r}} \sim 10^{-10} \mathrm{~cm}^{3} \mathrm{~s}^{-1116}$ and $k_{\text {Auger }}=3 \times 10^{-28} \mathrm{~cm}^{6}$ $\mathrm{s}^{-1117}$. Steady-state $n$ and $p$ values are then found for a given $I_{\text {exc,ASPL }}$.

To account for heat diffusion across the solid, the heat equation is simultaneously solved. Its one-dimensional form is used, given the rotational and translational (depth) symmetry of the system

$$
\frac{\mathrm{d} T(r)}{\mathrm{d} t}=D_{\mathrm{a}} \nabla^{2} T+\frac{Q_{\text {net }}}{\rho_{\mathrm{a}} C_{\mathrm{a}}} .
$$

In Eq. 34, $D_{\mathrm{a}}=10^{-4} \mathrm{~cm}^{2} \mathrm{~s}^{-1118}$ is the aerogel heat diffusivity, $\rho_{\mathrm{a}}=0.1 \mathrm{~g} \mathrm{~cm}^{-3118}$ is the aerogel density, and $C_{\mathrm{a}}$ $=2 \mathrm{Jg}^{-1} \mathrm{~K}^{-1118}$ is its specific heat.

$Q_{\text {net }}$ is given by

$$
Q_{\text {net }}=k_{\text {Auger }} n p^{2} E_{\mathrm{g}}-G^{\prime} \Delta E,
$$

where the first term reflects the Auger-induced heating of the NC and the second captures its cooling through emission upconversion. Blackbody contributions from the surrounding environment are excluded for simplicity. Equation 34 is solved using a forward-time center-space finite difference approach, where time steps are determined using the Von Neumann stability criterion ${ }^{119}$. An identical approach involving the simultaneous solution of both charge generation/recombination and heat diffusion equations in a solid has previously been used to model the optical cooling of bulk GaAs ${ }^{120}$.

Figure $11 \mathrm{~b}$ shows results of the simulation, where the final steady-state temperature at various points along the disk radius have been plotted. At the disk center, a $\Delta T=$ $37 \mathrm{~K}$ cooling, starting from room temperature, is predicted, with a final steady-state temperature of $T=263 \mathrm{~K}$. The inset of Fig. 11b shows that the final disk center temperature is reached on a $0.5 \mathrm{~s}$ timescale. Towards the disk edges, temperatures progressively rise to $300 \mathrm{~K}$. Figure 11c shows a false color map of the aerogel's resulting steady-state temperature profile.

These results clearly indicate the feasibility of cooling $\mathrm{CsPbBr}_{3} \mathrm{NCs}$. Improvements that can be considered in future modeling and experimental studies include the use of better thermal isolation schemes to improve the predicted/achieved cooling floor. They also include optimizing NC concentrations to maximize the absorption of subgap photons while maintaining the thermal properties of the aerogel. Of additional note is that the simulation approach employed here is not exclusive to $\mathrm{CsPbBr}_{3}$ and can be applied to study the potential cooling of other promising nanostructures.

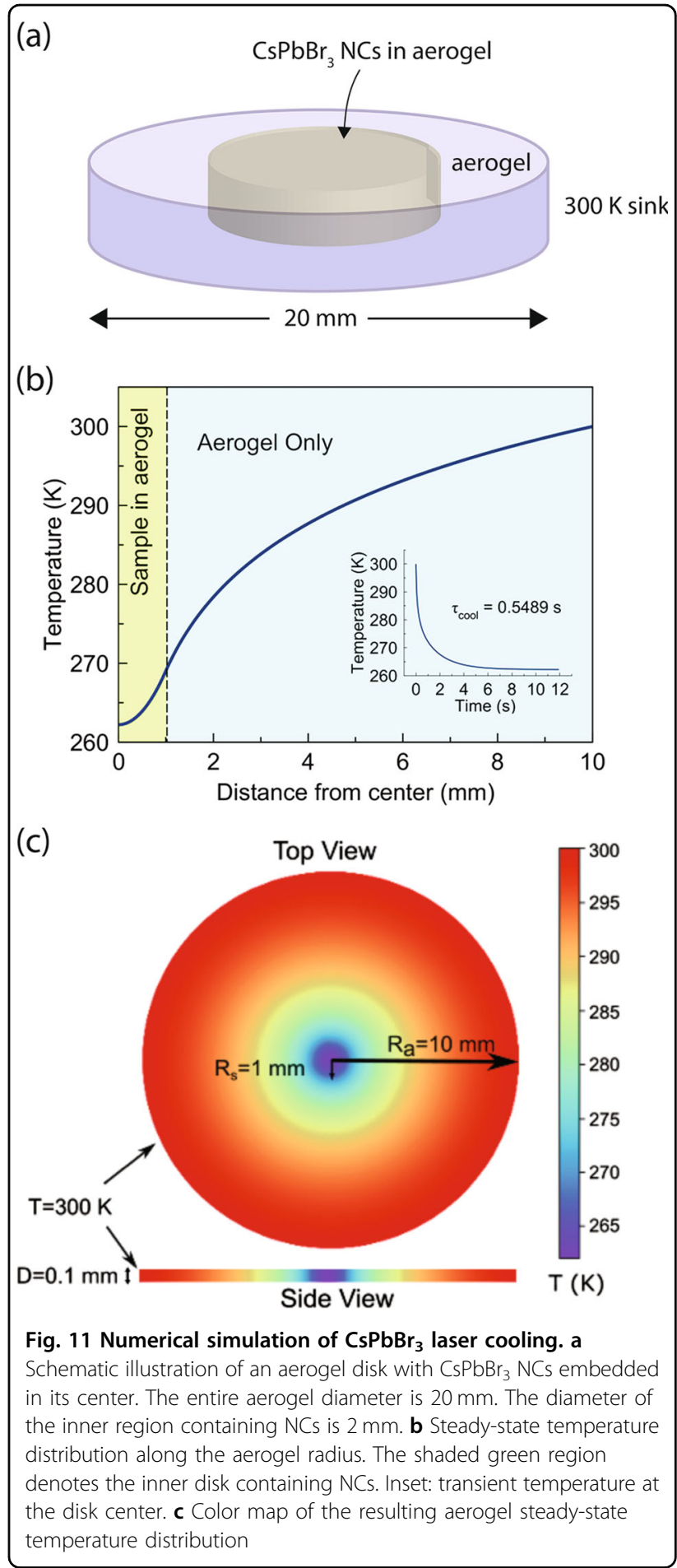

\section{Prior suggestions of nanostructure laser cooling}

Finally, before concluding, we discuss prior suggestions of $\mathrm{NC} /$ nanostructure laser cooling. In this regard, we are aware of only four reports suggesting to have successfully cooled a semiconductor. These are ref. ${ }^{47}$ (CdS NBs), ref. ${ }^{50}$ (hybrid perovskite nanoplatelets), and refs. ${ }^{51,52}$ (core/shell CdSe NCs). Concerns, however, exist 

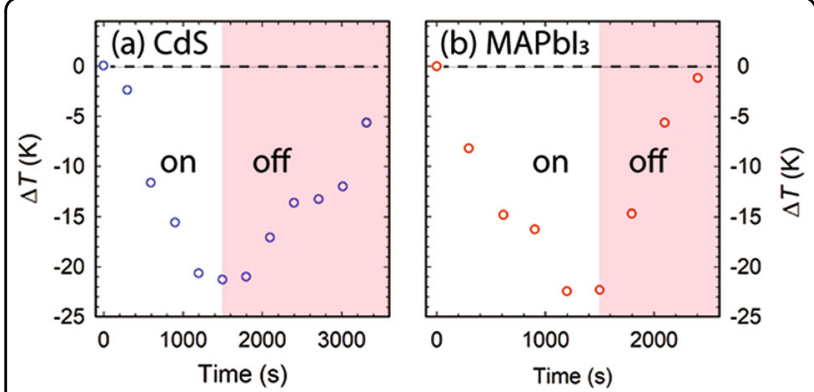

Fig. 12 Experimental laser cooling data from refs. ${ }^{47,50}$. a CdS NB $\left(\lambda_{\text {exc }}=532 \mathrm{~nm}\right)$ b MAPbl${ }_{3}$ crystal $\left(\lambda_{\text {exc }}=785 \mathrm{~nm}\right)$

regarding the validity of these studies, as outlined in ref. ${ }^{121}$ and below.

In the first two reports by Xiong and co-workers, a major discrepancy centers on the timescale over which individual $\mathrm{CdS} \mathrm{NBs}$ or hybrid perovskite platelets reportedly cool/heat. Specifically, Fig. 12a, b show extracted cooling and heating data from these studies and reveal that cooling occurs on a timescale of thousands of seconds. This is highlighted by the data in both figures, where the subgap excitation laser has been kept on. When $I_{\mathrm{exc}, \mathrm{ASPL}}=0$, both figures likewise reveal that nanostructure heating ensues over the course of $\sim 10^{3} \mathrm{~s}$.

However, numerical simulations, which consider charge generation/recombination and heat diffusion, predict that individual CdS NBs should cool (or heat) on a timescale of $\sim 10^{-4}$ s. In particular, an irradiated rectangular CdS cantilever (length of $20 \mu \mathrm{m}$, width of $5 \mu \mathrm{m}$, and thickness of $110 \mathrm{~nm}$ ), modeled in ref. ${ }^{107}$, cools (heats) over tens of microseconds. This is true for both 514 and $532 \mathrm{~nm}$ (below-gap) excitation. This cooling timescale easily differs by 7 orders of magnitude from what has been reported by Xiong and co-workers in Fig. 12. This discrepancy becomes even larger when a doubly clamped $\mathrm{CdS}$ cantilever is modeled.

An upper bound to the expected cooling timescale can be estimated for the case of a thermally isolated NB. Namely, an energy balance expression for its temperature change is

$$
\frac{V C_{\mathrm{p}} \mathrm{d}\left(T-T_{0}\right)}{\mathrm{d} t}=-V \alpha\left(\nu_{\mathrm{exc}}\right) I_{\mathrm{exc}} \eta_{\mathrm{ASPL}}-P_{\text {load }}
$$

where the first term on the right-hand side of the equation stems from upconversion-induced NB cooling. The second term reflects NB heating due to blackbody radiation from the surroundings. In Eq. 36, $V$ is the NB volume, $C_{\mathrm{p}}$ is its volumetric heat capacity, and $\alpha\left(v_{\mathrm{exc}}\right)$ is the sub-band gap absorption coefficient. The accompanying blackbody heating load can be expressed as

$$
P_{\text {load }}=A \sigma\left(T^{4}-T_{0}^{4}\right) \approx 4 A \sigma T_{0}^{3}\left(T-T_{0}\right),
$$

where $A$ is the total NB surface area and $\sigma$ is the Stefan-Boltzmann constant.

Solving Eq. 36 then yields

$$
T(t) \approx T_{0}-\frac{\alpha\left(\nu_{\mathrm{exc}}\right) I_{\mathrm{exc}} \eta_{\mathrm{ASPL}} \tau}{C_{\mathrm{p}}}\left(1-e^{-\frac{t}{\tau}}\right)
$$

and reveals a characteristic cooling/heating time constant of

$$
\tau=\frac{C_{p}}{4\left(\frac{A}{V}\right) \sigma T_{0}^{3}} .
$$

Upon introducing the following parameters for a CdS $\mathrm{NB}\left(C_{\mathrm{p}}=1.62 \times 10^{6} \mathrm{~J} \mathrm{~m}^{-3} \mathrm{~K}^{-1122}, \quad A=2.5 \times 10^{-6} \mathrm{~cm}^{2}\right.$, and $V=5 \times 10^{-11} \mathrm{~cm}^{347}$ ) one finds that $\tau=29 \mathrm{~ms}$. A perfectly isolated NB thus cools/heats on a timescale $\sim 4$ orders of magnitude faster than what has been reported in Fig. 12. A similar analysis can be conducted for hybrid perovskite nanoplatelets $^{50}$ to show that a large discrepancy in reported cooling/heating timescales also exists with this material. To illustrate, using the following parameters for $\mathrm{MAPbI}_{3}$ (a molar heat capacity of $C_{\mathrm{p}}=$ $9.7 \mathrm{~J} \mathrm{~K}^{-1} \mathrm{~mol}^{-1123}$, a corresponding density of $\rho_{\mathrm{a}}=$ $4.286 \mathrm{~g} \mathrm{~cm}^{-3124}$, and $\frac{A}{V}=\frac{1}{d}=5 \times 10^{4} \mathrm{~cm}^{-1}$, where the platelet thickness $d=200 \mathrm{~nm}^{50}$ ), Eq. 39 yields $\tau=2.2 \mathrm{~ms}$. This time constant is $\sim 6$ orders of magnitude smaller than the cooling/heating timescales in Fig. 12b.

In principle, slow cooling/heating timescales can stem from the presence of an additional thermal load in the system. For the NBs and nanoplatelets in question, this could arise from their respective $\mathrm{Si}$ or mica substrates. In fact, this scenario has been explored by Xiong and coworkers in ref. ${ }^{49}$, where the NB-induced cooling of a $\mathrm{SiO}_{2} / \mathrm{Si}$ substrate has been modeled. However, what has not been addressed in this study and in refs. ${ }^{47,48,50}$ is substrate heating due to the absorption of the incident light. A simple calculation for the NB case shows that $\sim 79 \%$ of the incident power will be absorbed by the $\mathrm{Si}$ substrate. Given its low emission quantum efficiency, subsequent nonradiative relaxation will heat both the substrate and the CdS NB. In this case, the estimated heating power is 50-fold greater than the reported NB cooling power. This suggests that cooling should not be observed. Together with the cooling/heating timescale discrepancies outlined above, this casts doubts on Xiong's suggestions of successful laser cooling.

Finally, Fontenot et al. ${ }^{51,52}$ has suggested that a suspension of commercial overcoated CdSe NCs can be macroscopically cooled. While intriguing, the QY of $80 \%$ reported by the manufacturer is below $\mathrm{QY}$ crit for CdSe in Table 1 and again implies that cooling should not be observed. Beyond this, other questions arise, as there are no details regarding the ASPL spectrum of the NCs or their $T$ and $\Delta E$ dependencies. There are also no estimates of $\eta_{\mathrm{ASPL}}$, which we have shown is critical to achieving 
condensed phase laser cooling. Last, there are concerns over the long-term temperature stability of the measurements despite the use of control specimens. Overall, it is our opinion that it is premature to suggest that condensed phase laser cooling has been achieved in any NC/nanostructure system to date.

\section{Outlook}

Six years ago, a purported breakthrough was reported, describing the optical cooling of individual $\mathrm{CdS} \mathrm{NBs}^{47}$. Although a debate now exists on whether cooling was actually achieved, the possibility that semiconductor nanostructures can be cooled has intrigued the community. This would capitalize on nearly three decades of research on their synthesis and optical characterization. Today, reports of unity or near-unity QYs in colloidal NCs coupled with the ability to upconvert light suggest the possibility of verifiably demonstrating condensed phase laser cooling. Although there remains much to be understood about $\mathrm{NC} /$ nanostructure upconversion, for example, the chemical identity of participating intermediate states as well as the apparent ubiquity of $\mathrm{NC} /$ nanostructure ASPL, it is clear that the field is within reach of realizing the century-old concept of optically cooling matter via anti-Stokes emission.

\section{Acknowledgements}

M.K. thanks the MURI:MARBLe project under the auspices of the Air Force Office of Scientific Research (Award No. FA9550-16-1-0362) for their financial support of this study.

\section{Conflict of interests}

The authors declare that have no conflict of interest.

\section{Publisher's note}

Springer Nature remains neutral with regard to jurisdictional claims in published maps and institutional affiliations.

Received: 18 April 2019 Revised: 3 July 2019 Accepted: 7 July 2019. Published online: 11 October 2019

\section{References}

1. Stokes, G. G. On the change of refrangibility of light. Philos. Trans. R. Soc. 142, 463-562 (1852).

2. Hansch, T. W. \& Schawlow, A. L. Cooling of gases by laser radiation. Opt. Commun. 13, 68-69 (1975).

3. Wineland, D. \& Dehmelt, H. Proposed $10^{14} \delta v<v$ laser fluorescence spectroscopy on $\mathrm{TI}+$ mono-ion oscillator II (spontaneous quantum jumps). Bull. Am. Phys. Soc. 20, 637 (1975).

4. Phillips, W. D. \& Metcalf, H. Laser deceleration of an atomic beam. Phys. Rev. Lett. 48, 596-599 (1982).

5. Chu, S., Hollberg, L., Bjorkholm, J. E., Cable, A. \& Ashkin, A. Three-dimensional viscous confinement and cooling of atoms by resonance radiation pressure. Phys. Rev. Lett. 55, 48-51 (1985).

6. Aspect, A., Arimondo, E., Kaiser, R., Vansteenkiste, N. \& Cohen-Tannoudji, C. Laser cooling below the one-photon recoil energy by velocityselective coherent population trapping. Phys. Rev. Lett. 61, 826-829 (1988).
7. Anderson, M. H., Ensher, J. R., Matthews, M. R., Wieman, C. E. \& Cornell, E. A. Observation of Bose-Einstein condensation in a dilute atomic vapor. Science 269, 198-201 (1995).

8. Davis, K. B. et al. Bose-Einstein condensation in a gas of sodium atoms. Phys. Rev. Lett. 75, 3969-3973 (1995).

9. Pringsheim, P. Zwei bemerkungen über den unterschied von lumineszenzund temperaturstrahlung. Z. Phys. 57, 739-746 (1929).

10. Epstein, R. I., Buchwald, M. I., Edwards, B. C., Gosnell, T. R. \& Mungan, C. E. Observation of laser-induced fluorescent cooling of a solid. Nature 377, 500-503 (1995).

11. Mungan, C. E., Buchwald, M. I., Edwards, B. C., Epstein, R. I. \& Gosnell, T. R. Laser cooling of a solid by $16 \mathrm{~K}$ starting from room temperature. Phys. Rev. Lett. 78 1030-1033 (1997).

12. Luo, X., Eisaman, M. D. \& Gosnell, T. R. Laser cooling of a solid by $21 \mathrm{~K}$ starting from room temperature. Opt. Lett. 23, 639-641 (1998).

13. Gosnell, T. R. Laser cooling of a solid by $65 \mathrm{~K}$ starting from room temperature. Opt. Lett. 24, 1041-1043 (1999).

14. Thiede, J., Distel, J., Greenfield, S. R. \& Epstein, R. I. Cooling to 208 K by optical refrigeration. Appl. Phys. Lett. 86, 154107 (2005).

15. Melgaard, S. D., Seletskiy, D. V., Di Lieto, A., Tonelli, M. \& Sheik-Bahae, M. Optical refrigeration to $119 \mathrm{~K}$, below National Institute of Standards and Technology cryogenic temperature. Opt. Lett. 38, 1588-1590 (2013).

16. Melgaard, S. D., Albrecht, A. R., Hehlen, M. P. \& Sheik-Bahae, M. Solid-state optical refrigeration to sub-100 Kelvin regime. Sci. Rep. 6, 20380 (2016).

17. Hehlen, M. P. et al. First demonstration of an all-solid-state optical cryocooler. Light Sci. Appl. 7, 15 (2018).

18. Roder, P. B., Smith, B. E., Zhou, X., Crane, M. J. \& Pauzauskie, P. J. Laser refrigeration of hydrothermal nanocrystals in physiological media. Proc. Natl. Acad. Sci. USA 112, 15024-15029 (2015).

19. Seletskiy, D. V. et al. Laser cooling of solids to cryogenic temperatures. Nat. Photonics 4, 161-164 (2010).

20. Rupper, G., Kwong, N. H. \& Binder, R. Large excitonic enhancement of optical refrigeration in semiconductors. Phys. Rev. Lett. 97, 117401 (2006).

21. Bender, D. A., Cederberg, J. G. Wang, C. \& Sheik-Bahae, M. Development of high quantum efficiency GaAs/GalnP double heterostructures for laser cooling. Appl. Phys. Lett. 102, 252102 (2013).

22. Imangholi, B., Hasselbeck, M. P., Sheik-Bahae, M., Epstein, R. I. \& Kurtz, S. Effects of epitaxial lift-off on interface recombination and laser cooling in GalnP/ GaAs heterostructures. Appl. Phys. Lett. 86, 081104 (2005).

23. Seletskiy, D. V., Epstein, R. I. \& Sheik-Bahae, M. Laser cooling in solids: advances and prospects. Rep. Prog. Phys. 79, 096401 (2016).

24. Gaponenko, S. V. Optical Properties of Semiconductor Nanocrystals 1st edn (Cambridge University Press, Cambridge, UK, 1998).

25. Yoffe, A. D. Semiconductor quantum dots and related systems: electronic, optical, luminescence and related properties of low dimensional systems. Adv. Phys. 50, 1-208 (2001).

26. Mungan, C. E. \& Gosnell, T. R. Laser cooling of solids. Adv. At. Mol. Opt. Phys. 40, 161-228 (1999).

27. Rayner, A., Heckenberg, N. R. \& Rubinsztein-Dunlop, H. Condensed-phase optical refrigeration. J. Opt. Soc. Am. B 20, 1037-1053 (2003).

28. Sheik-Bahae, M. \& Epstein, R. I. Optical refrigeration. Nat. Photonics 1, 693-699 (2007).

29. Sheik-Bahae, M. \& Epstein, R. I. Laser cooling of solids. Laser Photon. Rev. $\mathbf{3}$ 67-84 (2009).

30. Epstein, R. I. \& Sheik-Bahae, M. Optical Refrigeration: Science and Appli-cations of Laser cooling of Solids (WILEY-VCH Verlag GmbH \& Co. KGaA, Weinheim, Germany, 2009).

31. Petrushkin, S. \& Samartsev, V. Laser Cooling of Solids (Woodhead Publishing, Cambridge, UK, 2009).

32. Amirtharaj, P. M. \& Seiler, D. G. in Handbook of Optics, Vol. IV (ed. Bass, M.) (McGraw-Hill Inc., New York, 1995).

33. Tell, B., Damen, T. C. \& Porto, S. P. S. Raman effect in cadmium sulfide. Phys. Rev. 144, 771-774 (1966).

34. Lin, C., Kelley, D. F., Rico, M. \& Kelley, A. M. The "surface optical" phonon in CdSe nanocrystals. ACS Nano 8, 3928-3938 (2014).

35. Ninomiya, S. \& Adachi, S. Optical properties of cubic and hexagonal CdSe. J. Appl. Phys. 78, 4681-4689 (1995).

36. Sheik-Bahae, M. \& Epstein, R. I. Can laser light cool semiconductors? Phys. Rev. Lett. 92, 247403 (2004) 
37. Wang, J. B., Ding, D., Johnson, S. R., Yu, S. Q. \& Zhang, Y. H. Determination and improvement of spontaneous emission quantum efficiency in GaAs/AlGaAs heterostructures grown by molecular beam epitaxy. Phys. Stat. Sol. B 244 2740-2751 (2007).

38. Gauck, H., Gfroerer, T. H., Renn, M. J., Cornell, E. A. \& Bertness, K. A. External radiative quantum efficiency of $96 \%$ from a GaAs/GalnP heterostructure. Appl. Phys. A 64, 143-147 (1997).

39. Kuno, M. Colloidal quantum dots: a model nanoscience system. J. Phys. Chem. Lett. 4, 680-680 (2013).

40. Murray, C. B., Norris, D. J. \& Bawendi, M. G. Synthesis and characterization of nearly monodisperse $\mathrm{CdE}$ ( $E=$ sulfur, selenium, tellurium) semiconductor nanocrystallites. J. Am. Chem. Soc. 115, 8706-8715 (1993).

41. Empedocles, S. A., Neuhauser, R. \& Bawendi, M. G. Three-dimensional orientation measurements of symmetric single chromophores using polarization microscopy. Nature 399, 126-130 (1999).

42. Wehrenberg, B. L., Wang, C. \& Guyot-Sionnest, P. Interband and intraband optical studies of PbSe colloidal quantum dots. J. Phys. Chem. B 106, 10634-10640 (2002).

43. Protesescu, L. et al. Nanocrystals of cesium lead halide perovskites ( $\mathrm{CsPbX}_{3}$, $\mathrm{X}=\mathrm{Cl}, \mathrm{Br}$, and I): novel optoelectronic materials showing bright emission with wide color gamut. Nano Lett. 15, 3692-3696 (2015).

44. Hines, M. A. \& Guyot-Sionnest, P. Synthesis and characterization of strongly luminescing ZnS-capped CdSe nanocrystals. J. Phys. Chem. 100, 468-471 (1996).

45. Dabbousi, B. O. et al. (CdSe)ZnS core-shell quantum dots: synthesis and characterization of a size series of highly luminescent nanocrystallites. J. Phys. Chem. B 101, 9463-9475 (1997).

46. Peng, X., Schlamp, M. C., Kadavanich, A. V. \& Alivisatos, A. P. Epitaxial growth of highly luminescent $\mathrm{CdSe} / \mathrm{CdS}$ core/shell nanocrystals with photostability and electronic accessibility. J. Am. Chem. Soc. 119, 7019-7029 (1997).

47. Zhang, J., Li, D., Chen, R. \& Xiong, Q. Laser cooling of a semiconductor by 40 kelvin. Nature 493, 504-508 (2013).

48. Li, D., Zhang, J. \& Xiong, Q. Laser cooling of CdS nanobelts: thickness matters. Opt. Express 21, 19302-19310 (2013).

49. Li, D., Zhang, J., Wang, X., Huang, B. \& Xiong, Q. Solid-state semiconductor optical cryocooler based on CdS nanobelts. Nano Lett. 14, 4724-4728 (2014).

50. Ha, S.-T., Shen, C., Zhang, J. \& Xiong, Q. Laser cooling of organic-inorganic lead halide perovskites. Nat. Photonics 10, 115-121 (2016).

51. Fontenot, R. S., Mathur, V. K., Barkyoumb, J. H., Mungan, C. E. \& Tran, T. N. Measuring the anti-stokes luminescence of CdSe/ZnS quantum dots for laser cooling applications. Proc. SPIE 9821, 982103 (2016).

52. Fontenot, R. S., Mathur, V. K. \& Barkyoumb, J. H. New photothermal deflection technique to discriminate between heating and cooling. J. Quant. Spect. Rad. Trans. 204, 1-6 (2018).

53. Abazovic, N. D. et al. Photoluminescence of anatase and rutile $\mathrm{TiO}_{2}$ particles. J. Phys. Chem. B 110, 25366-25370 (2006).

54. Abazovic, N. D. et al. Photon energy up-conversion in colloidal $\mathrm{TiO}_{2}$ nanorods. Opt. Mater. 30, 1139-1144 (2008).

55. Sercel, P. C., Efros, A. L. \& Rosen, M. Intrinsic gap states in semiconductor nanocrystals. Phys. Rev. Lett. 83, 2394 (1999).

56. Ezhov, A. A. et al. Liquid-crystalline polymer composites with CdS nanorods: structure and optical properties. Langmuir 27, 13353-13360 (2011).

57. Morozov, Y. V. et al. Defect-mediated CdS nanobelt photoluminescence upconversion. J. Phys. Chem. C 121, 16607-16616 (2017).

58. Poles, E., Selmarten, D. C., Micic, O. I. \& Nozik, A. J. Anti-Stokes photoluminescence in colloidal semiconductor quantum dots. J. Chem. Phys. 75, 224708 (1999).

59. Rakovich, Y. P. et al. Anti-stokes photoluminescence in II-VI colloidal nanocrystals. Phys. Stat. Sol. B 229, 449-452 (2002).

60. Rakovich, Y. P. et al. Up-conversion luminescence via a below-gap state in CdSe/ZnS quantum dots. Phys. E Low. Dimens. Syst. Nanostruct. 17, 99-100 (2003).

61. Rusakov, K. I. et al. Control of efficiency of photon energy up-conversion in CdSe/ZnS quantum dots. Opt. Spectrosc. 94, 859-863 (2003).

62. $\mathrm{Li}, \mathrm{X}$. et al. Photoluminescence up-conversion of $\mathrm{CdSe} / \mathrm{ZnS}$ core/shell quantum dots under high pressure. J. Phys. Chem. C 113, 4737-4740 (2009).

63. Rakovich, Y. P. \& Gladyshchuk, A. Anti-stokes luminescence of cadmium telluride nanocrystals. J. Appl. Spectrosc. 69, 383-387 (2002).

64. Filonovich, S. A. et al. Up-conversion luminescence in colloidal CdTe nanocrystals. MRS Proc. 737, E13.12 (2002).
65. Wang, $X$. et al. Photoluminescence upconversion in colloidal CdTe quantum dots. Phys. Rev. B. 68, 125318 (2003).

66. Ragab, A. E., Gadallah, A. -S., Mohamed, M. B. \& Azzouz, I. M. Photoluminescence and upconversion on $\mathrm{Ag} / \mathrm{CdTe}$ quantum dots. Opt. Laser Technol. 63, 8-12 (2014).

67. Xiong, Y., Liu, C., Wang, J., Han, J. \& Zhao, X. Near-infrared anti-Stokes photoluminescence of $\mathrm{PbS}$ QDs embedded in glasses. Opt. Express 25, 6874-6882 (2017).

68. Zhang, Y. et al. Near-infrared-emitting colloidal $\mathrm{Ag}_{2} \mathrm{~S}$ quantum dots exhibiting upconversion luminescence. Superlattices Microstruct. 102, 512-516 (2017).

69. Ye, S. et al. Mechanistic investigation of upconversion photoluminescence in all-inorganic perovskite $\mathrm{CsPbBrl}_{2}$ nanocrystals. J. Phys. Chem. C 122, 3152-3156 (2018).

70. Ouyang, J. et al. Upconversion luminescence of colloidal CdS and ZnCdS semiconductor quantum dots. J. Phys. Chem. C. 111, 16261-16266 (2007).

71. Wu, W. Z. et al. Upconversion luminescence of CdTe nanocrystals by use of near-infrared femtosecond laser excitation. Opt. Lett. 32, 1174-1176 (2007).

72. Fernee, M. J., Jensen, P. \& Rubinsztein-Dunlop, H. Unconventional photoluminescence upconversion from PbS quantum dots. Appl. Phys. Lett. 91, 043112 (2007)

73. Roman, B. J. \& Sheldon, M. The role of mid-gap states in all-inorganic CsPbBr nanoparticle one photon up-conversion. Chem. Commun. 54, 6851-6854 (2018).

74. Seidel, W., Titkov, A., Andre, J. P., Voisin, P. \& Voos, M. High-efficiency energy up-conversion by an "Auger-Fountain" at an InP-AllnAs type-Il heterojunction. Phys. Rev. Lett. 73, 2356-2359 (1994).

75. Diener, J. et al. Strong low-temperature anti-stokes photoluminescence from coupled silicon nanocrystals. Opt. Mater. 17, 135-139 (2001).

76. Pelant, I. \& Valenta, J. Luminescence Spectroscopy of Semiconductors (Oxford Univ. Press, Oxford, 2012).

77. Vietmeyer, F., Frantsuzov, P. A., Janko, B. \& Kuno, M. Carrier recombination dynamics in individual CdSe nanowires. Phys. Rev. B 83, 115319 (2011).

78. Kuno, M., Lee, J. K., Dabbousi, B. O., Mikulec, F. V. \& Bawendi, M. G. The band edge luminescence of surface modified CdSe nanocrystallites: probing the luminescing state. J. Chem. Phys. 106, 9869-9882 (1997).

79. Brennan, M. C. et al. Origin of the size-dependent stokes shift in $\mathrm{CsPbBr}_{3}$ perovskite nanocrystals. J. Am. Chem. Soc. 139, 12201-12208 (2017).

80. Würth, C., Grabolle, M., Pauli, J., Spieles, M. \& Resch-Genger, U. Comparison of methods and achievable uncertainties for the relative and absolute measurement of photoluminescence quantum yields. Anal. Chem. 83, 3431-3439 (2011).

81. Resch-Genger, U. \& Rurack, K. Determination of the photoluminescence quantum yield of dilute dye solutions (IUPAC Technical Report). Pure Appl. Chem. 85, 2005-2026 (2013).

82. Würth, C., Grabolle, M., Pauli, J., Spieles, M. \& Resch-Genger, U. Relative and absolute determination of fluorescence quantum yields of transparent samples. Nat. Protoc. 8, 1535-1550 (2013).

83. Brouwer, A. M. Standards for photoluminescence quantum yield measurements in solution (IUPAC Technical Report). Pure Appl. Chem. 83, 2213-2228 (2011).

84. Greben, M., Fucikova, A. \& Valenta, J. Photoluminescence quantum yield of PbS nanocrystals in colloidal suspensions. J. Appl. Phys. 117, 144306 (2015).

85. Grabolle, M. et al. Determination of the fluorescence quantum yield of quantum dots: Suitable procedures and achievable uncertainties. Anal. Chem. 81, 6285-6294 (2009).

86. Laverdant, J. et al. Experimental determination of the fluorescence quantum yield of semiconductor nanocrystals. Materials 4, 1182-1193 (2011).

87. Würth, C., Geißler, D. \& Resch-Genger, U. Quantification of anisotropy-related uncertainties in relative photoluminescence quantum yield measurements of nanomaterials - semiconductor quantum dots and rods. Z. Phys. Chem. 229, 153-165 (2015).

88. Geißler, D., Würth, C., Wolter, C., Weller, H. \& Resch-Genger, U. Excitation wavelength dependence of the photoluminescence quantum yield and decay behavior of CdSe/CdS quantum dot/quantum rods with different aspect ratios. Phys. Chem. Chem. Phys. 19, 12509-12516 (2017).

89. Resch-Genger, U. et al. Traceability in fluorometry: Part II. Spectral fluorescence standards. J. Fluoresc. 15, 315-336 (2005).

90. Ahn, T.-S., Al-Kaysi, R. O., Müller, A. M., Wentz, K. M. \& Bardeen, C. J. Selfabsorption correction for solid-state photoluminescence quantum yields 
obtained from integrating sphere measurements. Rev. Sci. Instrum. 78, 086105 (2007).

91. Wang, C. et al. GaAs/GalnP double heterostructure characterization for laser cooling of semiconductors. Proc. SPIE 7951, 79510D (2011).

92. Wang, C. \& Sheik-Bahae, M. Determination of external quantum efficiency in semiconductors using pulsed power-dependent photoluminescence. Opt. Eng. 56, 011106 (2016).

93. Draguta, S. et al. Spatially non-uniform trap state densities in solutionprocessed hybrid perovskite thin films. J. Phys. Chem. Lett. 7, 715-721 (2016).

94. Imangholi, B. Investigation of Laser Cooling in Semiconductors. Ph.D. thesis, The University of New Mexico, Albuquerque (2006).

95. Robel, I., Gresback, R., Kortshagen, U., Schaller, R. D. \& Klimov, V. I. Universal size-dependent trend in auger recombination in direct-gap and indirect-gap semiconductor nanocrystals. Phys. Rev. Lett. 102, 177404 (2009).

96. Klimov, V. I., Mikhailovsky, A. A., McBranch, D. W., Leatherdale, C. A. \& Bawendi, M. G. Quantization of multiparticle auger rates in semiconductor quantum dots. Science 287, 1011-1013 (2000).

97. Tomasini, E. P., San Román, E. \& Braslavsky, S. E. Validation of fluorescence quantum yields for light-scattering powdered samples by laser-induced optoacoustic spectroscopy. Langmuir 25, 5861-5868 (2009).

98. Olmsted, J. Calorimetric determinations of absolute fluorescence quantum yields. J. Phys. Chem. 83, 2581-2584 (1979).

99. Santhi, A. et al. Thermal lens technique to evaluate the fluorescence quantum yield of a schiff base. Appl. Phys. B 79, 629-633 (2004).

100. Wang, C., Li, C.-Y., Hasselbeck, M. P., Imangholi, B. \& Sheik-Bahae, M. Precision, all-optical measurement of external quantum efficiency in semiconductors. J. Appl. Phys. 109, 093108 (2011)

101. Swarnkar, A. et al. Colloidal $\mathrm{CsPbBr}_{3}$ perovskite nanocrystals: luminescence beyond traditional quantum dots. Angew. Chem. Int. Ed. 54, 15424-15428 (2015).

102. Harbold, J. M. \& Wise, F. W. Photoluminescence spectroscopy of PbSe nanocrystals. Phys. Rev. B 76, 125304 (2007)

103. Morozov, Y. V., Zhang, S., Brennan, M. C., Janko, B. \& Kuno, M. Photoluminescence up-conversion in $\mathrm{CsPBr}_{3}$ nanocrystals. ACS Energy Lett. 2, 2514-2515 (2017).

104. Jakubek, Z. J., DeVries, J., Lin, S., Ripmeester, J. \& Yu, K. Exciton recombination and unconverted photoluminescence in colloidal CdSe quantum dots. J. Phys. Chem. C 112, 8153-8158 (2008).

105. Rakovich, Y. P., Jäckel, F., Donegan, J. F. \& Rogach, A. L. Semiconductor nanowires self-assembled from colloidal CdTe nanocrystal building blocks: optical properties and application perspectives. J. Mater. Chem. 22, 20831-20839 (2012).

106. Ye, S., Yu, M., Zhao, M., Song, J. \& Qu, J. Low temperature synthesis of highquality all-inorganic cesium lead halide perovskite nanocrystals in open air and their upconversion luminescence. J. Alloy. Compd. 730, 62-70 (2018).

107. Pant, A. et al. Optomechanical thermometry of nanoribbon cantilevers. J. Phys. Chem. C 112, 7525-7532 (2018).

108. Zhang, Q. et al. Phonon-assisted anti-stokes lasing in ZnTe nanoribbons. Adv. Mater. 28, 276-283 (2016).

109. Chen, W., Joly, A. G. \& McCready, D. E. Upconversion luminescence from CdSe nanoparticles. J. Chem. Phys. 122, 224708 (2005).

110. $\mathrm{Wu}, \mathrm{W}$. et al. Upconversion luminescent characteristics and peak shift of CdSeS nanocrystals under different wavelength laser excitation. J. Nanopart. Res. 13, 1049-1061 (2011).

111. Wu, W., Yu, D., Ye, H., Gao, Y. \& Chang, Q. Temperature and composition dependent excitonic luminescence and exciton-phonon coupling in CdSeS nanocrystals. Nanoscale Res. Lett. 7, 301 (2012).

112. Ananthakumar, S. et al. Size-independent peak shift between normal and upconversion photoluminescence in MPA-capped CdTe nanoparticles. Pramana - J. Phys. 82, 353-358 (2014).

113. Ananthakumar, S. et al. Size dependence of upconversion photoluminescence in MPA capped CdTe quantum dots: existence of upconversion bright point. J. Lumin. 169, 308-312 (2016).

114. Sun, G., Chen, R., Ding, Y. J. \& Khurgin, J. B. Upconversion due to opticalphonon-assisted anti-Stokes photoluminescence in bulk GaN. ACS Photonics 2, 628-632 (2015).

115. Loranger, S. et al. Spectroscopic and life-time measurements of quantum dot doped glass for optical refrigeration: a feasibility study. Proc. SPIE 8638, 863801 (2013)
116. Zhu, H. et al. Organic cations might not be essential to the remarkable properties of band edge carriers in lead halide perovskites. Adv. Mater. 29, 1603072 (2017).

117. Makarov, N. S. et al. Spectral and dynamical properties of single excitons, biexcitons, and trions in cesium-lead-halide perovskite quantum dots. Nano Lett. 16, 2349-2362 (2016).

118. Scheuerpflug, P., Hauck, M. \& Fricke, J. Thermal properties of silica aerogels between 1.4 and 330 K. J. Non-Cryst. Solids 145, 196-201 (1992).

119. Burden, R. L. \& Faires, J. D. Numerical Analysis 9th edn (Cengage Learning Boston, 2015).

120. Morozov, Y. V., Zhang, S., Janko, B. \& Kuno, M. Charge and thermal modeling of a semiconductor-based optical refrigerator. Appl. Phys. Lett. 113, 181105 (2018).

121. Morozov, Y. V. et al. Can lasers really refrigerate CdS nanobelts? Nature $\mathbf{5 7 0}$ E60-E61 (2019).

122. Madelung, O., Rössler, U. \& Schulz, M. II-VI and I-VII Compounds; Semimagnetic Compounds (Springer, Berlin, Germany, 1999).

123. Onoda-Yamamuro, N., Matsuo, T. \& Suga, H. Calorimetric and IR spectroscopic studies of phase transitions in methylammonium trihalogenoplumbates (II). J. Phys. Chem. Solids 51, 1383-1395 (1990).

124. Baikie, T. et al. Synthesis and crystal chemistry of the hybrid perovskite $\left(\mathrm{CH}_{3} \mathrm{NH}_{3}\right) \mathrm{Pbl}_{3}$ for solid-state sensitised solar cell applications. J. Mater. Chem. A 1, 5628-5641 (2013).

125. Yu, W. W., Qu, L., Guo, W. \& Peng, X. Experimental determination of the extinction coefficient of $\mathrm{CdTe}$, CdSe, and CdS nanocrystals. Chem. Mater. 15 2854-2860 (2003).

126. Weidman, M. C., Beck, M. E., Hoffman, R. S., Prins, F. \& Tisdale, W. A. Monodisperse, air-stable PbS nanocrystals via precursor stoichiometry control. ACS Nano 8, 6363-6371 (2014).

127. Dai, Q. et al. Size-dependent composition and molar extinction coefficient of PbSe semiconductor nanocrystals. ACS Nano 3, 1518-1524 (2009).

128. Maes, J. et al. Light absorption coefficient of $\mathrm{CsPbBr}_{3}$ perovskite nanocrystals. J. Phys. Chem. Lett. 9, 3093-3097 (2018).

129. Iaru, C. M., Geuchies, J. J., Koenraad, P. M., Vanmaekelbergh, D. \& Silov, A. Y. Strong carrier-phonon coupling in lead halide perovskite nanocrystals. ACS Nano 11, 11024-11030 (2017).

130. Swarnkar, A. et al. Quantum dot-induced phase stabilization of a-CsPbl perovskite for high-efficiency photovoltaics. Science 354, 92-95 (2016).

131. Gao, Y. \& Peng, X. Photogenerated excitons in plain core CdSe nanocrystals with unity radiative decay in single channel: the effects of surface and ligands. J. Am. Chem. Soc. 137, 4230-4235 (2015).

132. Greytak, A. B. et al. Alternating layer addition approach to $\mathrm{CdSe} / \mathrm{CdS}$ core/ shell quantum dots with near-unity quantum yield and high on-time fractions. Chem. Sci. 3, 2028-2034 (2012).

133. Saha, A. et al. Near-unity quantum yield in semiconducting nanostructures: structural understanding leading to energy efficient applications. J. Phys. Chem. Lett. 4, 3544-3549 (2013).

134. Chen, O. et al. Compact high-quality CdSe-CdS core-shell nanocrystals with narrow emission linewidths and suppressed blinking. Nat. Mater. 12, 445-451 (2013).

135. Pu, C. \& Peng, X. To battle surface traps on $\mathrm{CdSe} / \mathrm{CdS}$ core/shell nanocrystals: shell isolation versus surface treatment. J. Am. Chem. Soc. 138, 8134-8142 (2016).

136. Coropceanu, I., Rossinelli, A., Caram, J. R., Freyria, F. S. \& Bawendi, M. G. Slowinjection growth of seeded $\mathrm{CdSe} / \mathrm{CdS}$ nanorods with unity fluorescence quantum yield and complete shell to core energy transfer. ACS Nano 10, 3295-3301 (2016).

137. Koscher, B. A., Swabeck, J. K, Bronstein, N. D. \& Alivisatos, A. P. Essentially trapfree $\mathrm{CsPbBr}_{3}$ colloidal nanocrystals by postsynthetic thiocyanate surface treatment. J. Am. Chem. Soc. 139, 6566-6569 (2017).

138. Li, G. et al. Surface ligand engineering for near-unity quantum yield inorganic halide perovskite QDs and high performance QLEDs. Chem. Mater. 30 6099-6107 (2018).

139. Liu, F. et al. Highly luminescent phase-stable $\mathrm{CSPbl}_{3}$ perovskite quantum dots achieving near $100 \%$ absolute photoluminescence quantum yield. ACS Nano 11, 10373-10383 (2017).

140. Dai, S.-W. et al. Perovskite quantum dots with near unity solution and neatfilm photoluminescent quantum yield by novel spray synthesis. Adv. Mater. 30, 1705532 (2018).

141. Amani, M. et al. Near-unity photoluminescence quantum yield in $\mathrm{MoS}_{2}$. Science 350, 1065-1068 (2015). 